\title{
OPERATOR INTEGRALS WITH RESPECT TO A SPECTRAL MEASURE AND SOLUTIONS TO SOME OPERATOR EQUATIONS
}

\author{
SERGIO ALBEVERIO AND ALEXANDER K. MOTOVILOV
}

\begin{abstract}
We introduce the concept of Stieltjes integral of an operator-valued function with respect to the spectral measure associated with a normal operator. We give sufficient conditions for the existence of this integral and find bounds on its norm. The results obtained are applied to the Sylvester and Riccati operator equations. Assuming that the entry $C$ is a normal operator, the spectrum of the entry $A$ is separated from the spectrum of $C$, and $D$ is a bounded operator, we obtain a representation for the strong solution $X$ to the Sylvester equation $X A-C X=D$ in the form of an operator Stieltjes integral with respect to the spectral measure of $C$. By using this result we then establish sufficient conditions for the existence of a strong solution to the operator Riccati equation $Y A-C Y+Y B Y=D$ where $B$ is another bounded operator.
\end{abstract}

\section{INTRODUCTION}

In this paper we deal with integrals formally written as

$$
\int_{\Omega} F(z) d E(z) \text { or } \int_{\Omega} d E(z) G(z)
$$

where $\Omega$ is a Borel subset of the complex plane $\mathbb{C}$ and $E(\cdot)$ the spectral measure associated with a normal operator on a Hilbert space $\mathfrak{K}$. It is assumed that $F$ is an operator-valued function on $\Omega$ with values in the space $\mathscr{B}(\mathfrak{K}, \mathfrak{H})$ of bounded operators from $\mathfrak{K}$ to another Hilbert space $\mathfrak{H}$. Similarly, $G$ is assumed to be an operator-valued function mapping $\Omega$ into $\mathscr{B}(\mathfrak{H}, \mathfrak{K})$. Clearly, a reasonable definition of integration in 1.1 should yield operators from $\mathfrak{K}$ to $\mathfrak{H}$ and from $\mathfrak{H}$ to $\mathfrak{K}$, respectively.

The integrals of the form (1.1) are of interest in itself. But they also arise in many applications, in particular in the study of spectral subspace perturbation problems (see, e.g., [1]). Such integrals appear to be a useful tool in the study of the Sylvester and Riccati operator equations (see [2, $4,22,25]$ ).

There is an important particular case where $F(z)$ is given by

$$
F(z)=\varphi(A, z) T
$$

with $\varphi(\zeta, z)$ a sufficiently nice scalar function of two complex variables $\zeta$ and $z, A$ another normal operator on $\mathfrak{H}$, and $T$ a bounded operator from $\mathfrak{K}$ to $\mathfrak{H}$. In this case the integral $\int_{\Omega} F(z) d E(z)$ can be understood as a double operator Stieltjes integral [7]. More precisely,

$$
\int_{\Omega} F(z) d E(z)=\int_{\widetilde{\Omega}} \int_{\Omega} \varphi(\zeta, z) d \widetilde{E}(\zeta) T d E(z)
$$

Date: May 08, 2005.

1991 Mathematics Subject Classification. Primary 47A56, 47A62; Secondary 47B15, 47B49.

Key words and phrases. Operator Stieltjes integral, operator-valued function, normal operator, spectral measure, Sylvester equation, Riccati equation. 
where $\widetilde{E}(\cdot)$ is the spectral measure associated with $A$ and $\widetilde{\Omega}=\operatorname{spec}(A)$. Mainly due to the works by M. Sh. Birman and M. Z. Solomyak [8, 10] (see also references cited in [7]) there exists already a rather comprehensive theory of the double operator Stieltjes integrals.

However, this is not the case with more general integrals of the form 1.1. the ones that contain operator-valued functions $F(z)$ and $G(z)$ which cannot be written in terms of functions of normal operators like in (1.2). To the best of our knowledge, there is a well established approach to operator integrals of the type (1.1) only in the case where the spectral measure $E(\cdot)$ is associated with a self-adjoint operator (see [1, 3] 4, 22], and references therein). In particular, in [1] it was proven that the integrals (1.1) make sense as Riemann-Stieltjes integrals whenever $\Omega$ is a finite interval on the real axis and the operatorvalued functions $F$ and $G$ are Lipschitz on $\Omega$. Moreover, in this case the integrals (1.1) exist in the sense of the uniform operator topology. Some sufficient conditions for the existence of the improper integrals (1.1) in the case where the spectral measure $E(\cdot)$ corresponds to an unbounded self-adjoint operator are given in [4] 22].

The present paper is aimed at extending the main concepts and results of the operator Stieltjes integration theory to the case where the spectral measure $E(\cdot)$ may correspond to an arbitrary normal operator. Actually, we consider the integrals 1.1 in a somewhat more general setup, admitting the operator-valued functions $F(\lambda, \mu)$ and $G(\lambda, \mu)$ of two real variables $\lambda=\operatorname{Re} z$ and $\mu=\operatorname{Im} z$, i.e. functions that may depend on both $z$ and its conjugate $\bar{z}$. The integrals (1.1) are introduced in the usual way as limits (if they exist) of the corresponding Riemann-Stieltjes integral sums as the norm of a partition approaches zero (see Definition 2.2). Our main result is as follows (see Theorem 2.5).

Assume that $\Omega$ is a finite rectangle in $\mathbb{C}$ and $F(\lambda, \mu)$ is a $\mathscr{B}(\mathfrak{K}, \mathfrak{H})$-valued Lipschitz function defined for $\lambda+\mathrm{i} \mu \in \Omega$, that is, there is a $\gamma_{1}>0$ such that

$$
\begin{gathered}
\left\|F(\lambda, \mu)-F\left(\lambda^{\prime}, \mu^{\prime}\right)\right\| \leq \gamma_{1}\left(\left|\lambda-\lambda^{\prime}\right|+\left|\mu-\mu^{\prime}\right|\right) \\
\text { whenever } \lambda+\mathrm{i} \mu \in \Omega \text { and } \lambda^{\prime}+\mathrm{i} \mu^{\prime} \in \Omega .
\end{gathered}
$$

If, in addition, for some $\gamma_{2}>0$ the function $F$ satisfies the condition

$$
\begin{gathered}
\left\|F(\lambda, \mu)-F\left(\lambda^{\prime}, \mu\right)-F\left(\lambda, \mu^{\prime}\right)+F\left(\lambda^{\prime}, \mu^{\prime}\right)\right\| \leq \gamma_{2}\left|\lambda-\lambda^{\prime}\right|\left|\mu-\mu^{\prime}\right| \\
\text { whenever } \lambda+\mathrm{i} \mu \in \Omega \text { and } \lambda^{\prime}+\mathrm{i} \mu^{\prime} \in \Omega,
\end{gathered}
$$

then the operator Stieltjes integral

$$
\int_{\Omega} F(\operatorname{Re} z, \operatorname{Im} z) d E(z)
$$

exists in the sense of the uniform operator topology. The same statement also holds (see Corollary 2.77 for an operator Stieltjes integral of the form

$$
\int_{\Omega} d E(z) G(\operatorname{Re} z, \operatorname{Im} z)
$$

whenever $G$ is a $\mathscr{B}(\mathfrak{H}, \mathfrak{K})$-valued function on $\Omega$ satisfying 1.4 and 1.5 .

This result allows us to extend the concept of an operator Stieltjes integral with respect to the spectral measure of a normal operator $C$ to the case where $\Omega=\operatorname{spec}(C)$, by taking into account that only the values of $F(\lambda, \mu)$ and $G(\lambda, \mu)$ for $\lambda+\mathrm{i} \mu \in \operatorname{spec}(C)$ contribute to (1.6) and (1.7), respectively. By using this concept we then obtain an integral representation for the solution $X$ to the operator Sylvester equation

$$
X A-C X=D,
$$

where $A$ is a closed densely defined possibly unbounded operator on a Hilbert space $\mathfrak{H}, C$ a possibly unbounded normal operator on a Hilbert space $\mathfrak{K}$, and $D \in \mathscr{B}(\mathfrak{H}, \mathfrak{K})$. In particular, 
under the assumption that

$$
\operatorname{dist}(\operatorname{spec}(A), \operatorname{spec}(C))>0
$$

we prove that $X \in \mathscr{B}(\mathfrak{H}, \mathfrak{K})$ is a unique strong solution to (1.8) if and only if it can be represented in the form of the operator Stieltjes integral

$$
X=\int_{\operatorname{spec}(C)} d E_{C}(z) D(A-z)^{-1},
$$

which converges in the sense of the strong operator topology (see Theorem 4.5). So far, such a representation was only proven in the case where $C$ is a self-adjoint operator (see [4] Theorem 2.14]).

We apply the results obtained also to the operator Riccati equation

$$
X A-C X+X B X=D,
$$

where $B \in \mathscr{B}(\mathfrak{K}, \mathfrak{H})$ and the entries $A, C$, and $D$ satisfy the same assumptions as in (1.8). If $X \in \mathscr{B}(\mathfrak{H}, \mathfrak{K})$ is a strong solution to 1.11 such that $\operatorname{spec}(\operatorname{spec}(A+B X), \operatorname{spec}(C))>0$, by using (1.10) one writes the Riccati equation in the equivalent integral form

$$
X=\int_{\operatorname{spec}(C)} d E_{C}(z) D(A+B X-z)^{-1} .
$$

Under the assumption (1.9) and additional "smallness" assumptions upon $B$ and $D$ we prove the existence of a solution $X \in \mathscr{B}(\mathfrak{H}, \mathfrak{K})$ to the integral equation (1.12). This solution to (1.12) also solves the Riccati equation (1.11) (see Theorem 5.7).

The plan of the paper is as follows.

In Section 2 we introduce the concept of a Stieltjes integral of an operator-valued function with respect the spectral measure of a normal operator and prove the main result (Theorem 2.5) concerning sufficient conditions for the existence of such integrals. We also derive a norm estimate (Lemma 2.9) for these integrals.

In Section 3 we extend the concept [4, 22] of the norm of a bounded operator with respect to a spectral measure to the case where this measure is associated with a normal operator.

In Section 4 we discuss the Sylvester equation 1.8. In particular, we prove that any weak solution to this equation is also a strong solution. This result allows us to present refined versions of the representation theorems [4, Section 2] for the solution $X$ of the operator Silvester equation, extending the integral representations for $X$ to the case where the entry $C$ in 1.8 is a normal operator.

Finally, in Section 5 we prove the above mentioned existence result (Theorem 5.7) for the Riccati equation 1.11 .

We conclude the introduction with the description of some more notations that are used throughout the paper. The identity operator on any Hilbert space $\mathfrak{K}$ is denoted by $I$. If $T$ is a closed operator on $\mathfrak{K}$, by $\operatorname{spec}(T)$ we always denote the spectrum of $T$. We will also use the notation

$$
\sigma(T)=\left\{(\lambda, \mu) \in \mathbb{R}^{2} \mid \lambda+\mathrm{i} \mu \in \operatorname{spec}(T)\right\}
$$

for the natural imbedding of $\operatorname{spec}(T)$ into the real plane $\mathbb{R}^{2}$. The set

$$
\mathscr{W}(T)=\{z \in \mathbb{C} \mid z=\langle T x, x\rangle \text { for some } x \in \operatorname{Dom}(T),\|x\|=1\}
$$

is called the numerical range of the operator $T$. 


\section{INTEGRAL OF AN OPERATOR-VALUED FUNCTION WITH RESPECT TO THE SPECTRAL MEASURE OF A NORMAL OPERATOR}

Let $\mathscr{A}_{\text {Borel }}(\mathbb{C})$ denote the algebra of Borel subsets of the complex plane $\mathbb{C}$ and let $\{E(\Omega)\}_{\Omega \in \mathscr{A}_{\text {Borel }}(\mathbb{C})}$ be the spectral family of a (possibly unbounded) normal operator $C$. Recall that $E(\Omega)$ 's are orthogonal projections in $\mathfrak{K}$ that possess the properties (see, e.g., 6 $\S 5.1])$

$$
\begin{aligned}
& E\left(\Omega^{\prime} \cap \Omega^{\prime \prime}\right)=E\left(\Omega^{\prime}\right) E\left(\Omega^{\prime \prime}\right) \text { for any } \Omega^{\prime}, \Omega^{\prime \prime} \in \mathscr{A}_{\text {Borel }}(\mathbb{C}), \\
& E\left(\Omega^{\prime} \cup \Omega^{\prime \prime}\right)=E\left(\Omega^{\prime}\right)+E\left(\Omega^{\prime \prime}\right) \text { whenever } \Omega^{\prime}, \Omega^{\prime \prime} \in \mathscr{A}_{\text {Borel }}(\mathbb{C}) \text { and } \Omega^{\prime} \cap \Omega^{\prime \prime}=\varnothing,
\end{aligned}
$$

and

$$
E(\mathbb{C})=E(\operatorname{spec}(C))=I .
$$

With the spectral family $\{E(\Omega)\}_{\Omega \in \mathscr{A}_{\text {Borel }}(\mathbb{C})}$ by

$$
\mathrm{E}(\Lambda)=E\left(\left\{z=\lambda+\mathrm{i} \mu \mid(\lambda, \mu) \in \Lambda \subset \mathbb{R}^{2}\right\}\right)
$$

we associate the projection-valued measure

$$
\{\mathrm{E}(\Lambda)\}_{\Lambda \in \mathscr{A}_{\text {Borel }}\left(\mathbb{R}^{2}\right)}
$$

on the algebra $\mathscr{A}_{\text {Borel }}\left(\mathbb{R}^{2}\right)$ of Borel subsets of $\mathbb{R}^{2}$. Clearly, 2.1), (2.2), and (2.3) are equivalent to

$$
\begin{aligned}
& \mathrm{E}\left(\Lambda^{\prime} \cap \Lambda^{\prime \prime}\right)=\mathrm{E}\left(\Lambda^{\prime}\right) \mathrm{E}\left(\Lambda^{\prime \prime}\right) \text { for any } \Lambda^{\prime}, \Lambda^{\prime \prime} \in \mathscr{A}_{\text {Borel }}\left(\mathbb{R}^{2}\right) \\
& \mathrm{E}\left(\Lambda^{\prime} \cup \Lambda^{\prime \prime}\right)=\mathrm{E}\left(\Lambda^{\prime}\right)+\mathrm{E}\left(\Lambda^{\prime \prime}\right) \text { whenever } \Lambda^{\prime}, \Lambda^{\prime \prime} \in \mathscr{A}_{\text {Borel }}\left(\mathbb{R}^{2}\right) \text { and } \Lambda^{\prime} \cap \Lambda^{\prime \prime}=\varnothing
\end{aligned}
$$

and

respectively.

$$
E\left(\mathbb{R}^{2}\right)=E(\sigma(C))=I
$$

In terms of the spectral measures $E(\cdot)$ and $E(\cdot)$, we write the spectral decomposition of the normal operator $C$ either as

$$
C=\int_{\mathbb{C}} z d E(z)=\int_{\operatorname{spec}(C)} z d E(z)
$$

or as

$$
C=\int_{\mathbb{R}^{2}}(\lambda+\mathrm{i} \mu) d \mathrm{E}(\lambda, \mu)=\int_{\sigma(C)}(\lambda+\mathrm{i} \mu) d \mathrm{E}(\lambda, \mu) .
$$

Further, we introduce the projection-valued function $\widehat{\mathrm{E}}(\lambda, \mu)$ on $\mathbb{R}^{2}$ by

$$
\widehat{\mathrm{E}}(\lambda, \mu)=\mathrm{E}\left(\left\{(x, y) \in \mathbb{R}^{2} \mid x<\lambda, y<\mu\right\}\right) .
$$

In the following the function $\widehat{\mathrm{E}}(\lambda, \mu)$ is called the spectral function of the normal operator $C$. In contrast to the case of self-adjoint operators the spectral function of a normal operator is a function of two real variables.

Clearly, for $\lambda \leq \lambda^{\prime}$ and $\mu \leq \mu^{\prime}$ the additivity property of a spectral measure implies

$\widehat{\mathrm{E}}\left(\lambda^{\prime}, \mu^{\prime}\right)=\widehat{\mathrm{E}}(\lambda, \mu)+\mathrm{E}\left((-\infty, \lambda) \times\left[\mu, \mu^{\prime}\right)\right)+\mathrm{E}\left(\left[\lambda, \lambda^{\prime}\right) \times(-\infty, \mu)\right)+\mathrm{E}\left(\left[\lambda, \lambda^{\prime}\right) \times\left[\mu, \mu^{\prime}\right)\right)$ and hence

$$
\widehat{\mathrm{E}}(\lambda, \mu) \leq \widehat{\mathrm{E}}\left(\lambda^{\prime}, \mu^{\prime}\right) \text { if } \lambda \leq \lambda^{\prime} \text { and } \mu \leq \mu^{\prime},
$$

that is, $\widehat{E}(\lambda, \mu)$ is a non-decreasing function in both variables $\lambda$ and $\mu$.

One also observes that if $\lambda \leq \lambda^{\prime}$ and $\mu \leq \mu^{\prime}$ then

$$
\widehat{\mathrm{E}}\left(\lambda^{\prime}, \mu^{\prime}\right)-\widehat{\mathrm{E}}\left(\lambda^{\prime}, \mu\right)-\widehat{\mathrm{E}}\left(\lambda, \mu^{\prime}\right)+\widehat{\mathrm{E}}(\lambda, \mu)=E\left(\left[\lambda, \lambda^{\prime}\right) \times\left[\mu, \mu^{\prime}\right)\right)
$$


and thus

$$
\widehat{\mathrm{E}}\left(\lambda^{\prime}, \mu\right)-\widehat{\mathrm{E}}(\lambda, \mu) \leq \widehat{\mathrm{E}}\left(\lambda^{\prime}, \mu^{\prime}\right)-\widehat{\mathrm{E}}\left(\lambda, \mu^{\prime}\right)
$$

for any $\mu, \mu^{\prime} \in \mathbb{R}$ such that $\mu \leq \mu^{\prime}$ and any $\lambda, \lambda^{\prime} \in \mathbb{R}$ such that $\lambda \leq \lambda^{\prime}$.

We remark that 2.4 implies

$$
\left\|\widehat{\mathrm{E}}\left(\lambda^{\prime}, \mu^{\prime}\right) x\right\| \leq\|\widehat{\mathrm{E}}(\lambda, \mu) x\| \text { for any } x \in \mathfrak{K} \text { whenever } \lambda^{\prime} \leq \lambda \text { and } \mu^{\prime} \leq \mu .
$$

Indeed

$$
\|\widehat{\mathrm{E}}(\lambda, \mu) x\|^{2}=\langle\widehat{\mathrm{E}}(\lambda, \mu) x, \widehat{\mathrm{E}}(\lambda, \mu) x\rangle=\langle\widehat{\mathrm{E}}(\lambda, \mu) x, x\rangle
$$

and

$$
\left\|\widehat{\mathrm{E}}\left(\lambda^{\prime}, \mu^{\prime}\right) x\right\|^{2}=\left\langle\widehat{\mathrm{E}}\left(\lambda^{\prime}, \mu^{\prime}\right) x, \widehat{\mathrm{E}}\left(\lambda^{\prime}, \mu^{\prime}\right) x\right\rangle=\left\langle\widehat{\mathrm{E}}\left(\lambda^{\prime}, \mu^{\prime}\right) x, x\right\rangle .
$$

Hence (2.7) is a consequence of (2.4).

By using the fact that both right-hand and left-hand sides of (2.6) are orthogonal projections, in a similar way one concludes that

$$
\begin{gathered}
\left\|\left(\widehat{\mathrm{E}}\left(\lambda^{\prime}, \mu\right)-\widehat{\mathrm{E}}(\lambda, \mu)\right) x\right\| \leq\left\|\left(\widehat{\mathrm{E}}\left(\lambda^{\prime}, \mu^{\prime}\right)-\widehat{\mathrm{E}}\left(\lambda, \mu^{\prime}\right)\right) x\right\| \\
\quad \text { for any } x \in \mathfrak{K} \text { whenever } \mu \leq \mu^{\prime} \text { and } \lambda \leq \lambda^{\prime} .
\end{gathered}
$$

For notational setup we adopt the following

Hypothesis 2.1. Let $\mathfrak{H}$ and $\mathfrak{K}$ be Hilbert spaces, $\Delta=[a, b) \times[c, d)$ a rectangle in $\mathbb{R}^{2}$ with $-\infty<a<b<+\infty$ and $-\infty<c<d<+\infty$. Let $\{\mathrm{E}(\Lambda)\}_{\Lambda \in \mathscr{A}_{\mathrm{Borel}}\left(\mathbb{R}^{2}\right)}$ be the spectral family associated with a normal operator on $\mathfrak{K}$.

Definition 2.2. Assume Hypothesis 2.1 An operator-valued function

$$
F: \Delta \rightarrow \mathscr{B}(\mathfrak{K}, \mathfrak{H})
$$

is said to be uniformly (resp. strongly, weakly) integrable from the right with respect the spectral measure $d \mathrm{E}(\lambda, \mu)$ on $\Delta$ if the limit

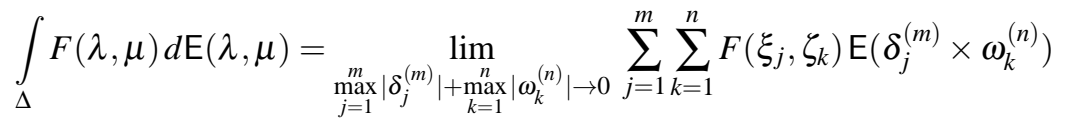

exists in the uniform (resp. strong, weak) operator topology. Here, $\delta_{j}^{(m)}=\left[\lambda_{j-1}, \lambda_{j}\right)$, $j=1,2, \ldots, m$, and $\omega_{k}^{(n)}=\left[\mu_{k-1}, \mu_{k}\right), k=1,2, \ldots, n$, where $a=\lambda_{0}<\lambda_{1}<\ldots<\lambda_{m}=b$ and $c=\mu_{0}<\mu_{1}<\ldots<\mu_{n}=b$ are partitions of the intervals $[a, b)$ and $[c, d)$, respectively; $\xi_{j} \in$ $\delta_{j}^{(m)}$ and $\zeta_{k} \in \delta_{k}^{(n)}$ are arbitrarily chosen points, $\left|\delta_{k}^{(j)}\right|=\lambda_{j}-\lambda_{j-1}$ and $\left|\omega_{k}^{(n)}\right|=\mu_{k}-\mu_{k-1}$. The limit value (2.9), if it exists, is called the right Stieltjes integral of the operator-valued function $F$ with respect to the measure $d \mathrm{E}(\lambda, \mu)$ on $\Delta$.

Similarly, a function

$$
G: \Delta \rightarrow \mathscr{B}(\mathfrak{H}, \mathfrak{K})
$$

is said to be uniformly (resp. strongly, weakly) integrable from the left with respect to the measure $d \mathrm{E}(\lambda, \mu)$ on $\Delta$, if the limit

$$
\int_{\Delta} d \mathrm{E}(\lambda, \mu) G(\lambda, \mu)=\lim _{\substack{m a x \\ j=1}} \lim _{j=\max _{k=1}^{n}\left|\omega_{k}^{(n)}\right| \rightarrow 0} \sum_{j=1}^{m} \sum_{k=1}^{n} \mathrm{E}\left(\delta_{j}^{(m)} \times \omega_{k}^{(n)}\right) G\left(\xi_{j}, \zeta_{k}\right)
$$

exists in the uniform (resp. strong, weak) operator topology. The corresponding limit value (2.10), if it exists, is called the left Stieltjes integral of the operator-valued function $G$ with respect to the measure $d \mathrm{E}(\lambda, \mu)$ on $\Delta$. 
The following statement can be considered an extension of [22, Lemma 10.5] to the case of the spectral measure associated with a normal operator.

Lemma 2.3. Assume Hypothesis 2.1 Then an operator-valued function $F(\lambda, \mu)$,

$$
F: \Delta \rightarrow \mathscr{B}(\mathfrak{K}, \mathfrak{H}),
$$

is integrable in the weak (uniform) operator topology with respect to the measure $d \mathrm{E}(\lambda, \mu)$ on the rectangle $\Delta$ from the left if and only if the function $[F(\lambda, \mu)]^{*}$ is integrable in the weak (uniform) operator topology with respect to the measure $d \mathrm{E}(\lambda, \mu)$ on $\Delta$ from the right and then

$$
\left[\int_{\Delta} F(\lambda, \mu) d \mathrm{E}(\lambda, \mu)\right]^{*}=\int_{\Delta} d \mathrm{E}(\lambda, \mu)[F(\lambda, \mu)]^{*} .
$$

Proof. Like in the proof of Lemma 10.5 in [22], the assertion is proven by taking into account the continuity property of the involution $T \rightarrow T^{*}$ with respect to operator uniform and operator weak convergence in $\mathscr{B}(\mathfrak{K}, \mathfrak{H})$. It suffices to apply this property to the integral sums in 2.9) and 2.10.

Remark 2.4. Since the involution $T \rightarrow T^{*}$ is not continuous with respect to the strong convergence (see, e.g., [6 $\$ 2.5]$ ), the convergence of one of the integrals (2.11) in the strong operator topology in general only implies the convergence of the other one in the weak operator topology.

Some sufficient conditions for the integrability of an operator-valued function $F(\lambda, \mu)$ with respect to the spectral measure of a normal operator are described in the following statement.

Theorem 2.5. Assume Hypothesis 2.1 Suppose that for some $\gamma_{1}>0$ the operator-valued function $F: \Delta \rightarrow \mathscr{B}(\mathfrak{K}, \mathfrak{H})$ satisfies the Lipschitz condition

$$
\left\|F(\lambda, \mu)-F\left(\lambda^{\prime}, \mu^{\prime}\right)\right\| \leq \gamma_{1}\left(\left|\lambda-\lambda^{\prime}\right|+\left|\mu-\mu^{\prime}\right|\right)
$$

and for some $\gamma_{2}>0$ the condition

$$
\begin{gathered}
\left\|F(\lambda, \mu)-F\left(\lambda^{\prime}, \mu\right)-F\left(\lambda, \mu^{\prime}\right)+F\left(\lambda^{\prime}, \mu^{\prime}\right)\right\| \leq \gamma_{2}\left|\lambda-\lambda^{\prime}\right|\left|\mu-\mu^{\prime}\right| \\
\text { for any } \lambda, \lambda^{\prime} \in[a, b) \text { and } \mu, \mu^{\prime} \in[c, d) .
\end{gathered}
$$

Then the function $F$ is right-integrable on $\Delta$ with respect to the spectral measure $d \mathrm{E}(\lambda, \mu)$ in the sense of the uniform operator topology.

Proof. Let $\left\{\delta_{j}^{(m)}\right\}_{j=1}^{m}$ and $\left\{\omega_{k}^{(n)}\right\}_{k=1}^{n}$ be partitions of the intervals $[a, b)$ and $[c, d)$, respectively, and let $\Delta_{j k}^{(m, n)}=\delta_{k}^{(m)} \times \omega_{k}^{(n)}, j=1,2, \ldots, m, k=1,2, \ldots, n$. Assume that $\left\{\left(\xi_{j}, \zeta_{k}\right) \in\right.$ $\left.\Delta_{j k}^{(m, n)}, j=1,2, \ldots, m, k=1,2, \ldots, n\right\}$ and $\left\{\left(\xi_{j}^{\prime}, \zeta_{k}^{\prime}\right) \in \Delta_{j k}^{(m, n)}, j=1,2, \ldots, m, k=1,2, \ldots, n\right\}$ are two sets of points.

First, we prove that the limit 2.9) (if it exists) does not depend on the choice of the points $\left(\xi_{j}, \zeta_{k}\right)$ within the partition rectangles $\Delta_{j k}^{(m, n)}$.

Let

$$
J_{m, n}=\sum_{j=1}^{m} \sum_{k=1}^{n} F\left(\xi_{j}, \zeta_{k}\right) \mathrm{E}\left(\Delta_{j k}^{(m, n)}\right)
$$

and

$$
J_{m, n}^{\prime}=\sum_{j=1}^{m} \sum_{k=1}^{n} F\left(\xi_{j}^{\prime}, \zeta_{k}^{\prime}\right) \mathrm{E}\left(\Delta_{j k}^{(m, n)}\right) .
$$


Observe that

$$
\mathrm{E}\left(\Delta_{j k}^{(m, n)}\right)=\widehat{\mathrm{E}}\left(\lambda_{j}, \mu_{k}\right)-\widehat{\mathrm{E}}\left(\lambda_{j-1}, \mu_{k}\right)-\widehat{\mathrm{E}}\left(\lambda_{j}, \mu_{k-1}\right)+\widehat{\mathrm{E}}\left(\lambda_{j-1}, \mu_{k-1}\right)
$$

and hence

$$
\begin{aligned}
J_{m, n}-J_{m, n}^{\prime}=\sum_{j=1}^{m} \sum_{k=1}^{n}\left(\left[F\left(\xi_{j}, \zeta_{k}\right)-F\left(\xi_{j}^{\prime}, \zeta_{k}^{\prime}\right)\right]\right. \\
\times\left[\widehat{\mathrm{E}}\left(\lambda_{j}, \mu_{k}\right)-\widehat{\mathrm{E}}\left(\lambda_{j-1}, \mu_{k}\right)-\widehat{\mathrm{E}}\left(\lambda_{j}, \mu_{k-1}\right)+\widehat{\mathrm{E}}\left(\lambda_{j-1}, \mu_{k-1}\right)\right] .
\end{aligned}
$$

Represent the difference $J_{m, n}-J_{m, n}^{\prime}$ as the sum of two terms that are more convenient for estimating

$$
J_{m, n}-J_{m, n}^{\prime}=L_{1}+L_{2}
$$

where

$$
\begin{aligned}
L_{1}=\sum_{j=1}^{m} \sum_{k=1}^{n}( & {\left[F\left(\xi_{j}, \zeta_{k}\right)-F\left(\xi_{j}^{\prime}, \zeta_{k}\right)\right] } \\
& \times\left[\widehat{\mathrm{E}}\left(\lambda_{j}, \mu_{k}\right)-\widehat{\mathrm{E}}\left(\lambda_{j-1}, \mu_{k}\right)-\widehat{\mathrm{E}}\left(\lambda_{j}, \mu_{k-1}\right)+\widehat{\mathrm{E}}\left(\lambda_{j-1}, \mu_{k-1}\right)\right]
\end{aligned}
$$

and

$$
\begin{aligned}
L_{2}=\sum_{j=1}^{m} \sum_{k=1}^{n}( & {\left[F\left(\xi_{j}^{\prime}, \zeta_{k}\right)-F\left(\xi_{j}^{\prime}, \zeta_{k}^{\prime}\right)\right] } \\
& \times\left[\widehat{\mathrm{E}}\left(\lambda_{j}, \mu_{k}\right)-\widehat{\mathrm{E}}\left(\lambda_{j-1}, \mu_{k}\right)-\widehat{\mathrm{E}}\left(\lambda_{j}, \mu_{k-1}\right)+\widehat{\mathrm{E}}\left(\lambda_{j-1}, \mu_{k-1}\right)\right] .
\end{aligned}
$$

By inspection one verifies that

$$
L_{1}=S_{1}+S_{2}+S_{3},
$$

where

$$
\begin{aligned}
S_{1} & =\sum_{j=1}^{m}\left[F\left(\xi_{j}^{\prime}, \zeta_{1}\right)-F\left(\xi_{j}, \zeta_{1}\right)\right]\left[\widehat{\mathrm{E}}\left(\lambda_{j}, \mu_{0}\right)-\widehat{\mathrm{E}}\left(\lambda_{j-1}, \mu_{0}\right)\right], \\
S_{2} & =\sum_{j=1}^{m}\left[F\left(\xi_{j}, \zeta_{n}\right)-F\left(\xi_{j}^{\prime}, \zeta_{n}\right)\right]\left[\widehat{\mathrm{E}}\left(\lambda_{j}, \mu_{n}\right)-\widehat{\mathrm{E}}\left(\lambda_{j-1}, \mu_{n}\right)\right]
\end{aligned}
$$

and

$$
\begin{aligned}
S_{3}=\sum_{j=1}^{m} \sum_{k=1}^{n-1}[ & \left.F\left(\xi_{j}, \zeta_{k}\right)-F\left(\xi_{j}^{\prime}, \zeta_{k}\right)-F\left(\xi_{j}, \zeta_{k+1}\right)+F\left(\xi_{j}^{\prime}, \zeta_{k+1}\right)\right] \\
& \times\left[\widehat{\mathrm{E}}\left(\lambda_{j}, \mu_{k}\right)-\widehat{\mathrm{E}}\left(\lambda_{j-1}, \mu_{k}\right)\right] .
\end{aligned}
$$

Clearly, for any $x \in \mathfrak{K}$ by the Lipschitz property (2.12)

$$
\left\|S_{1} x\right\| \leq \gamma_{1} \sum_{j=1}^{m}\left|\delta_{j}^{(m)}\right|\left\|\left(\widehat{\mathrm{E}}\left(\lambda_{j}, \mu_{0}\right)-\widehat{\mathrm{E}}\left(\lambda_{j-1}, \mu_{0}\right)\right) x\right\|
$$

and hence

$$
\begin{aligned}
\left\|S_{1} x\right\| & \leq \gamma_{1}\left(\sum_{j=1}^{m}\left|\delta_{j}^{(m)}\right|^{2}\right)^{1 / 2}\left(\sum_{j=1}^{m}\left\langle\left(\widehat{\mathrm{E}}\left(\lambda_{j}, \mu_{0}\right)-\widehat{\mathrm{E}}\left(\lambda_{j-1}, \mu_{0}\right)\right) x, x\right\rangle\right)^{1 / 2} \\
& \leq \gamma_{1}\left(\max _{j=1}^{m}\left|\delta_{j}^{(m)}\right|\right)^{1 / 2} \sqrt{b-a}\left(\left\langle\left(\widehat{\mathrm{E}}\left(b, \mu_{0}\right)-\widehat{\mathrm{E}}\left(a, \mu_{0}\right)\right) x, x\right\rangle\right)^{1 / 2}
\end{aligned}
$$




$$
\leq \gamma_{1}\left(\max _{j=1}^{m}\left|\delta_{j}^{(m)}\right|\right)^{1 / 2} \sqrt{b-a}\|\widehat{\mathrm{E}}(b, c)-\widehat{\mathrm{E}}(a, c)\|\|x\|,
$$

since

$$
\begin{aligned}
\left\langle\left(\widehat{\mathrm{E}}\left(b, \mu_{0}\right)-\widehat{\mathrm{E}}\left(a, \mu_{0}\right)\right) x, x\right\rangle & =\left\langle\left(\widehat{\mathrm{E}}\left(b, \mu_{0}\right)-\widehat{\mathrm{E}}\left(a, \mu_{0}\right)\right)^{2} x, x\right\rangle \\
& =\left\langle\left(\widehat{\mathrm{E}}\left(b, \mu_{0}\right)-\widehat{\mathrm{E}}\left(a, \mu_{0}\right)\right) x,\left(\widehat{\mathrm{E}}\left(b, \mu_{0}\right)-\widehat{\mathrm{E}}\left(a, \mu_{0}\right)\right) x\right\rangle \\
& =\left\|\left(\widehat{\mathrm{E}}\left(b, \mu_{0}\right)-\widehat{\mathrm{E}}\left(a, \mu_{0}\right)\right) x\right\|^{2}
\end{aligned}
$$

and $\mu_{0}=c$.

In a similar way one shows that for any $x \in \mathfrak{K}$

$$
\left\|S_{2} x\right\| \leq \gamma_{1}\left(\max _{j=1}^{m}\left|\delta_{j}^{(m)}\right|\right)^{1 / 2} \sqrt{b-a}\|\widehat{\mathrm{E}}(b, d)-\widehat{\mathrm{E}}(a, d)\|\|x\| .
$$

Finally, by using (2.13) at the fist step, for any $x \in \mathfrak{K}$ one estimates $S_{3} x$ as follows:

$$
\begin{aligned}
\left\|S_{3} x\right\| \leq & \gamma_{2} \sum_{j=1}^{m} \sum_{k=1}^{n-1}\left|\delta_{j}^{(m)}\left\|\omega_{k}^{(n)} \mid\right\|\left(\widehat{\mathrm{E}}\left(\lambda_{j}, \mu_{k}\right)-\widehat{\mathrm{E}}\left(\lambda_{j-1}, \mu_{k}\right)\right) x \|\right. \\
& =\gamma_{2} \sum_{k=1}^{n-1}\left|\omega_{k}^{(n)}\right| \sum_{j=1}^{m}\left|\delta_{j}^{(m)}\right|\left\|\left(\widehat{\mathrm{E}}\left(\lambda_{j}, \mu_{k}\right)-\widehat{\mathrm{E}}\left(\lambda_{j-1}, \mu_{k}\right)\right) x\right\| \\
& \leq \gamma_{2} \sum_{k=1}^{n-1}\left|\omega_{k}^{(n)}\right|\left(\sum_{j=1}^{m}\left|\delta_{j}^{(m)}\right|^{2}\right)^{1 / 2}\left(\sum_{j=1}^{m}\left\langle\left(\widehat{\mathrm{E}}\left(\lambda_{j}, \mu_{k}\right)-\widehat{\mathrm{E}}\left(\lambda_{j-1}, \mu_{k}\right)\right) x, x\right\rangle\right)^{1 / 2} \\
& \leq \gamma_{2}(d-c)\left(\max _{j=1}^{m}\left|\delta_{j}^{(m)}\right|\right)^{1 / 2} \sqrt{b-a} \max _{k=1}^{n-1}\left(\left\langle\left(\widehat{\mathrm{E}}\left(b, \mu_{k}\right)-\widehat{\mathrm{E}}\left(a, \mu_{k}\right)\right) x, x\right\rangle\right)^{1 / 2} \\
& \leq \gamma_{2} \sqrt{b-a}(d-c)\left(\max _{j=1}^{m}\left|\delta_{j}^{(m)}\right|\right)^{1 / 2} \max _{k=1}^{n-1}\left\|\widehat{\mathrm{E}}\left(b, \mu_{k}\right)-\widehat{\mathrm{E}}\left(a, \mu_{k}\right)\right\|\|x\|
\end{aligned}
$$

by applying (2.20) (with $\mu_{0}$ replaced by $\mu_{k}$ ) at the last step. Obviously, by (2.6) for any $k=1,2, \ldots, n-1$

$$
\widehat{\mathrm{E}}\left(b, \mu_{k}\right)-\widehat{\mathrm{E}}\left(a, \mu_{k}\right) \leq \widehat{\mathrm{E}}\left(b, \mu_{n}\right)-\widehat{\mathrm{E}}\left(a, \mu_{n}\right)=\widehat{\mathrm{E}}(b, d)-\widehat{\mathrm{E}}(a, d)
$$

and we arrive at the following final estimate for $S_{3}$ :

$$
\left\|S_{3} x\right\| \leq \gamma_{2} \sqrt{b-a}(d-c)\left(\max _{j=1}^{m}\left|\delta_{j}^{(m)}\right|\right)^{1 / 2}\|\widehat{\mathrm{E}}(b, d)-\widehat{\mathrm{E}}(a, d)\|\|x\| .
$$

Combining (2.18), 2.19, 2.21), and (2.22) one concludes that for any $x \in \mathfrak{K}$

$$
\left\|L_{1} x\right\| \leq M_{1}\left(\max _{j=1}^{m}\left|\delta_{j}^{(m)}\right|\right)^{1 / 2} \sqrt{b-a}\|x\|,
$$

where

$$
M_{1}=\gamma_{1}\|\widehat{\mathrm{E}}(b, c)-\widehat{\mathrm{E}}(a, c)\|+\left(\gamma_{1}+\gamma_{2}(d-c)\right)\|\widehat{\mathrm{E}}(b, d)-\widehat{\mathrm{E}}(a, d)\| .
$$

In a similar way one proves that an analogous estimate holds for the term $L_{2}$ given by (2.17),

$$
\left\|L_{2} x\right\| \leq M_{2}\left(\max _{k=1}^{n}\left|\omega_{k}^{(n)}\right|\right)^{1 / 2} \sqrt{d-c}\|x\|
$$


where

$$
M_{2}=\gamma_{1}\|\widehat{\mathrm{E}}(a, d)-\widehat{\mathrm{E}}(a, c)\|+\left(\gamma_{1}+\gamma_{2}(b-a)\right)\|\widehat{\mathrm{E}}(b, d)-\widehat{\mathrm{E}}(b, c)\| .
$$

Combining (2.16), 2.23), and 2.24) proves that if the sum in (2.9) converges strongly (resp. weakly, in the operator norm topology) for some choice of the numbers $\left\{\xi_{j} \in\right.$ $\left.\delta_{j}^{(m)}\right\}_{j=1}^{m}$ and $\left\{\zeta_{k} \in \omega_{k}^{(n)}\right\}_{k=1}^{n}$, then it converges strongly (resp. weakly, in the operator norm topology) to the same limit for any other choice of these numbers, in particular this takes place for the choice where

$$
\xi_{j}=\lambda_{j-1}, \quad j=1,2, \ldots, m, \quad \text { and } \quad \zeta_{k}=\mu_{j-1}, \quad k=1,2, \ldots, n .
$$

It remains to prove that the double sequence of the operators $J_{m, n}$ given by 2.14 has a limit as $m \rightarrow \infty, n \rightarrow \infty, \max _{j=1}^{m}\left|\delta_{j}^{(m)}\right| \rightarrow 0$, and $\max _{k=1}^{n}\left|\omega_{k}^{(n)}\right| \rightarrow 0$.

Assume that there are two different partitions of the interval $[a, b)$ containing $m$ and $\widetilde{m}>$ $m$ subintervals, respectively, and two different partitions of the interval $[c, d)$ containing $n$ and $\tilde{n}>n$ subintervals, respectively Further, assume, without loss of generality, that all the points of the $m$-partition of the interval $[a, b)$ are the points of the $\widetilde{m}$-partition of $[a, b)$ and all the points of the $\widetilde{n}$-partition of the interval $[c, d)$ are the points of the $n$-partition of $[c, d)$. Denote by $\lambda_{j, s}, s=0,1, \ldots, p_{j}$, the points of the $\widetilde{m}$-partition of $[a, b)$ that belong to the interval $\left[\lambda_{j-1}, \lambda_{j}\right]$ and by $\mu_{k, t}, t=0,1, \ldots, q_{k}$, the points of the $\widetilde{n}$-partition of $[c, d)$ belonging to the interval $\left[\mu_{k-1}, \mu_{k}\right]$. By definition of partition subintervals we have

$$
\begin{aligned}
\lambda_{j-1} & =\lambda_{j, 0}<\lambda_{j, 1}<\ldots<\lambda_{j, p_{j}}=\lambda_{j}, \quad j=1,2, \ldots, m, \\
\mu_{k-1} & =\mu_{k, 0}<\mu_{k, 1}<\ldots<\mu_{k, q_{k}}=\mu_{k}, \quad k=1,2, \ldots, n .
\end{aligned}
$$

In the following we assume that one chooses the points $\xi_{j}, \zeta_{k}$ and $\xi_{j, s}, \zeta_{k, t}$ like in 2.25) and thus

$$
J_{m, n}=\sum_{j=1}^{m} \sum_{k=1}^{n} F\left(\lambda_{j-1}, \mu_{k-1}\right) \mathrm{E}\left(\Delta_{j k}^{(m, n)}\right)
$$

and

$$
J_{\widetilde{m}, \widetilde{n}}=\sum_{j=1}^{m} \sum_{k=1}^{n} \sum_{s=1}^{p_{j}} \sum_{t=1}^{q_{k}} F\left(\lambda_{j, s-1}, \mu_{k, t-1}\right) \mathrm{E}\left(\Delta_{j, s ; k, t}^{(\widetilde{m}, \widetilde{n})}\right)
$$

where

$$
\Delta_{j, s ; k, t}^{(\widetilde{m}, \widetilde{n})}=\left[\lambda_{j, s-1}, \lambda_{j, s}\right) \times\left[\mu_{k, t-1 ; k, t}\right) .
$$

By 2.15

$$
\begin{aligned}
J_{m, n}= & \sum_{j=1}^{m} \sum_{k=1}^{n} F\left(\lambda_{j-1}, \mu_{k-1}\right) \\
& \times\left[\widehat{\mathrm{E}}\left(\lambda_{j}, \mu_{k}\right)-\widehat{\mathrm{E}}\left(\lambda_{j-1}, \mu_{k}\right)-\widehat{\mathrm{E}}\left(\lambda_{j}, \mu_{k-1}\right)+\widehat{\mathrm{E}}\left(\lambda_{j-1}, \mu_{k-1}\right)\right]
\end{aligned}
$$

and

$$
\begin{aligned}
J_{\widetilde{m}, \widetilde{n}}= & \sum_{j=1}^{m} \sum_{k=1}^{n} \sum_{s=1}^{p_{j}} \sum_{t=1}^{q_{k}} F\left(\lambda_{j, s-1}, \mu_{k, t-1}\right) \\
& \times\left[\widehat{\mathrm{E}}\left(\lambda_{j, s}, \mu_{k, t}\right)-\widehat{\mathrm{E}}\left(\lambda_{j, s-1}, \mu_{k, t}\right)-\widehat{\mathrm{E}}\left(\lambda_{j, s}, \mu_{k, t-1}\right)+\widehat{\mathrm{E}}\left(\lambda_{j, s-1}, \mu_{k, t-1}\right)\right] .
\end{aligned}
$$

Taking into account 2.26) and (2.27) one verifies by inspection that

$$
J_{\widetilde{m}, \tilde{n}}-J_{m n}=\sum_{j=1}^{m} \sum_{k=1}^{n}\left(T_{j k}^{(1)}+T_{j k}^{(2)}+T_{j k}^{(3)}\right),
$$


where

$$
\begin{aligned}
T_{j k}^{(1)}= & \sum_{s=1}^{p_{j}-1}\left[F\left(\lambda_{j, s}, \mu_{k, 0}\right)-F\left(\lambda_{j, s-1}, \mu_{k, 0}\right)\right] \\
\quad \times & {\left[\widehat{\mathrm{E}}\left(\lambda_{j, p_{j}}, \mu_{k, q_{k}}\right)-\widehat{\mathrm{E}}\left(\lambda_{j, s}, \mu_{k, q_{k}}\right)-\widehat{\mathrm{E}}\left(\lambda_{j, p_{j}}, \mu_{k, 0}\right)+\widehat{\mathrm{E}}\left(\lambda_{j, s}, \mu_{k, 0}\right)\right] } \\
= & \sum_{s=1}^{p_{j}-1}\left[F\left(\lambda_{j, s}, \mu_{k-1}\right)-F\left(\lambda_{j, s-1}, \mu_{k-1}\right)\right] \\
& \times\left[\widehat{\mathrm{E}}\left(\lambda_{j}, \mu_{k}\right)-\widehat{\mathrm{E}}\left(\lambda_{j, s}, \mu_{k}\right)-\widehat{\mathrm{E}}\left(\lambda_{j}, \mu_{k-1}\right)+\widehat{\mathrm{E}}\left(\lambda_{j, s}, \mu_{k-1}\right)\right], \\
T_{j k}^{(2)}= & \sum_{t=1}^{q_{k}-1}\left[F\left(\lambda_{j, 0}, \mu_{k, t}\right)-F\left(\lambda_{j, 0}, \mu_{k, t-1}\right)\right] \\
\quad & \times\left[\widehat{\mathrm{E}}\left(\lambda_{j, p_{j}}, \mu_{k, q_{k}}\right)-\widehat{\mathrm{E}}\left(\lambda_{j, 0}, \mu_{k, q_{k}}\right)-\widehat{\mathrm{E}}\left(\lambda_{j, p_{j}}, \mu_{k, t}\right)+\widehat{\mathrm{E}}\left(\lambda_{j, 0}, \mu_{k, t}\right)\right] \\
= & \sum_{t=1}^{q_{k}-1}\left[F\left(\lambda_{j-1}, \mu_{k, t}\right)-F\left(\lambda_{j-1}, \mu_{k, t-1}\right)\right] \\
& \times\left[\widehat{\mathrm{E}}\left(\lambda_{j}, \mu_{k}\right)-\widehat{\mathrm{E}}\left(\lambda_{j-1}, \mu_{k}\right)-\widehat{\mathrm{E}}\left(\lambda_{j}, \mu_{k, t}\right)+\widehat{\mathrm{E}}\left(\lambda_{j-1}, \mu_{k, t}\right)\right],
\end{aligned}
$$

and

$$
\begin{aligned}
T_{j k}^{(3)}= & \sum_{s=1}^{p_{j}-1} \sum_{t=1}^{q_{k}-1}\left[F\left(\lambda_{j, s}, \mu_{k, t}\right)-F\left(\lambda_{j, s-1}, \mu_{k, t}\right)-F\left(\lambda_{j, s}, \mu_{k, t-1}\right)+F\left(\lambda_{j, s-1}, \mu_{k, t-1}\right)\right] \\
& \times\left[\widehat{\mathrm{E}}\left(\lambda_{j}, \mu_{k}\right)-\widehat{\mathrm{E}}\left(\lambda_{j, s}, \mu_{k}\right)-\widehat{\mathrm{E}}\left(\lambda_{j}, \mu_{k, t}\right)+\widehat{\mathrm{E}}\left(\lambda_{j, s}, \mu_{k, t}\right)\right]
\end{aligned}
$$

One also notes that

$$
\begin{aligned}
\sum_{k=1}^{n} T_{j k}^{(1)}=\sum_{s=1}^{p_{j}-1}\{ & {\left[F\left(\lambda_{j, s}, \mu_{n-1}\right)-F\left(\lambda_{j, s-1}, \mu_{n-1}\right)\right]\left[\widehat{\mathrm{E}}\left(\lambda_{j}, \mu_{n}\right)-\widehat{\mathrm{E}}\left(\lambda_{j, s}, \mu_{n}\right)\right] } \\
- & {\left[F\left(\lambda_{j, s}, \mu_{0}\right)-F\left(\lambda_{j, s-1}, \mu_{0}\right)\right]\left[\widehat{\mathrm{E}}\left(\lambda_{j}, \mu_{0}\right)-\widehat{\mathrm{E}}\left(\lambda_{j, s}, \mu_{0}\right)\right] } \\
+ & \sum_{k=1}^{n-1}\left[F\left(\lambda_{j, s}, \mu_{k-1}\right)-F\left(\lambda_{j, s-1}, \mu_{k-1}\right)-F\left(\lambda_{j, s}, \mu_{k}\right)+F\left(\lambda_{j, s-1}, \mu_{k}\right)\right] \\
& \left.\times\left[\widehat{\mathrm{E}}\left(\lambda_{j}, \mu_{k}\right)-\widehat{\mathrm{E}}\left(\lambda_{j, s}, \mu_{k}\right)\right]\right\}
\end{aligned}
$$

and

$$
\begin{aligned}
\sum_{j=1}^{m} T_{j k}^{(2)}=\sum_{t=1}^{q_{k}-1}\{[ & \left.F\left(\lambda_{m-1}, \mu_{k, t}\right)-F\left(\lambda_{m-1}, \mu_{k, t-1}\right)\right]\left[\widehat{\mathrm{E}}\left(\lambda_{m}, \mu_{k}\right)-\widehat{\mathrm{E}}\left(\lambda_{m}, \mu_{k, t}\right)\right] \\
-[ & \left.F\left(\lambda_{0}, \mu_{k, t}\right)-F\left(\lambda_{0}, \mu_{k, t-1}\right)\right]\left[\widehat{\mathrm{E}}\left(\lambda_{0}, \mu_{k}\right)-\widehat{\mathrm{E}}\left(\lambda_{0}, \mu_{k, t}\right)\right] \\
+ & \sum_{j=1}^{m-1}\left[F\left(\lambda_{j-1}, \mu_{k, t}\right)-F\left(\lambda_{j-1}, \mu_{k, t-1}\right)-F\left(\lambda_{j}, \mu_{k, t}\right)+F\left(\lambda_{j}, \mu_{k, t-1}\right)\right] \\
& \left.\times\left[\widehat{\mathrm{E}}\left(\lambda_{j}, \mu_{k}\right)-\widehat{\mathrm{E}}\left(\lambda_{j}, \mu_{k, t}\right)\right]\right\}
\end{aligned}
$$


By (2.12) and (2.13) the equality 2.34 implies that for any $x \in \mathfrak{K}$

$$
\begin{aligned}
\left\|\sum_{k=1}^{n} T_{j k}^{(1)} x\right\| \leq \sum_{s=1}^{p_{j}-1}\{ & \gamma_{1}\left|\lambda_{j, s}-\lambda_{j, s-1}\right|\left\|\left(\widehat{\mathrm{E}}\left(\lambda_{j}, \mu_{n}\right)-\widehat{\mathrm{E}}\left(\lambda_{j, s}, \mu_{n}\right)\right) x\right\| \\
& +\gamma_{1}\left|\lambda_{j, s}-\lambda_{j, s-1}\right|\left\|\left(\widehat{\mathrm{E}}\left(\lambda_{j}, \mu_{0}\right)-\widehat{\mathrm{E}}\left(\lambda_{j, s}, \mu_{0}\right)\right) x\right\| \\
& \left.+\sum_{k=1}^{n-1} \gamma_{2}\left|\lambda_{j, s}-\lambda_{j, s-1}\right|\left|\omega_{k}^{(n)}\right|\left\|\left(\widehat{\mathrm{E}}\left(\lambda_{j}, \mu_{k}\right)-\widehat{\mathrm{E}}\left(\lambda_{j, s}, \mu_{k}\right)\right) x\right\|\right\}
\end{aligned}
$$

and thus

$$
\begin{aligned}
& \left\|\sum_{k=1}^{n} T_{j k}^{(1)} x\right\| \text { leq } \gamma_{1}\left|\delta_{j}^{(m)}\right| \max _{s=1}^{p_{j}-1}\left\|\left(\widehat{\mathrm{E}}\left(\lambda_{j}, d\right)-\widehat{\mathrm{E}}\left(\lambda_{j, s}, d\right)\right) x\right\| \\
& \quad+\gamma_{1}\left|\delta_{j}^{(m)}\right| \max _{s=1}^{p_{j}-1}\left\|\left(\widehat{\mathrm{E}}\left(\lambda_{j}, c\right)-\widehat{\mathrm{E}}\left(\lambda_{j, s}, c\right)\right) x\right\| \\
& +\gamma_{2}\left|\delta_{j}^{(m)}\right|(d-c) \mid \max _{k=1}^{n-1} \max _{s=1}^{p_{j}-1}\left\|\left(\widehat{\mathrm{E}}\left(\lambda_{j}, \mu_{k}\right)-\widehat{\mathrm{E}}\left(\lambda_{j, s}, \mu_{k}\right)\right) x\right\|,
\end{aligned}
$$

since $\mu_{0}=c, \mu_{n}=d$,

$$
\sum_{s=1}^{p_{j}-1}\left|\lambda_{j, s}-\lambda_{j, s-1}\right|=\lambda_{j, p_{j-1}}-\lambda_{j-1}<\lambda_{j}-\lambda_{j-1}=\left|\delta_{j}^{(m)}\right|
$$

and $\sum_{k=1}^{n-1}\left|\omega_{k}^{(n)}\right|<d-c$. Obviously

$$
\left\|\left(\widehat{\mathrm{E}}\left(\lambda_{j}, \mu\right)-\widehat{\mathrm{E}}\left(\lambda_{j, s}, \mu\right)\right) x\right\| \leq\left\|\left(\widehat{\mathrm{E}}\left(\lambda_{j}, \mu\right)-\widehat{\mathrm{E}}\left(\lambda_{j-1}, \mu\right)\right) x\right\| \text { for any } \mu \in \mathbb{R}
$$

since

$$
\begin{array}{r}
\left\|\left(\widehat{\mathrm{E}}\left(\lambda_{j}, \mu\right)-\widehat{\mathrm{E}}\left(\lambda_{j, s}, \mu\right)\right) x\right\|^{2}=\left\langle\mathrm{E}\left(\left[\lambda_{j, s}, \lambda_{j}\right) \times(-\infty, \mu)\right) x, x\right\rangle \\
\leq\left\langle\mathrm{E}\left(\left[\lambda_{j-1}, \lambda_{j}\right) \times(-\infty, \mu)\right) x, x\right\rangle \\
=\left\|\left(\widehat{\mathrm{E}}\left(\lambda_{j}, \mu\right)-\widehat{\mathrm{E}}\left(\lambda_{j-1}, \mu\right)\right) x\right\|^{2} .
\end{array}
$$

Then by using (2.36) and 2.8) one infers from 2.35) that

$$
\left\|\sum_{k=1}^{n} T_{j k}^{(1)} x\right\| \leq\left[2 \gamma_{1}+\gamma_{2}(d-c)\right]\left|\delta_{j}^{(m)}\right|\left\|\left(\widehat{\mathrm{E}}\left(\lambda_{j}, d\right)-\widehat{\mathrm{E}}\left(\lambda_{j-1}, d\right)\right) x\right\| .
$$

Therefore

$$
\begin{aligned}
&\left\|\sum_{j=1}^{m} \sum_{k=1}^{n} T_{j k}^{(1)} x\right\| \leq {\left[2 \gamma_{1}+\gamma_{2}(d-c)\right] \sum_{j=1}^{m}\left|\delta_{j}^{(m)}\right|\left\|\left(\widehat{\mathrm{E}}\left(\lambda_{j}, d\right)-\widehat{\mathrm{E}}\left(\lambda_{j-1}, d\right)\right) x\right\| } \\
& \leq {\left[2 \gamma_{1}+\gamma_{2}(d-c)\right]\left(\sum_{j=1}^{m}\left|\delta_{j}^{(m)}\right|^{2}\right)^{1 / 2}\left(\sum_{j=1}^{m}\left\|\left(\widehat{\mathrm{E}}\left(\lambda_{j}, d\right)-\widehat{\mathrm{E}}\left(\lambda_{j-1}, d\right)\right) x\right\|^{2}\right)^{1 / 2} } \\
& \leq\left[2 \gamma_{1}+\gamma_{2}(d-c)\right]\left(\max _{j=1}^{m}\left|\delta_{j}^{(m)}\right|\right)^{1 / 2}\left(\sum_{j=1}^{m}\left|\delta_{j}^{(m)}\right|\right)^{1 / 2} \\
& \times\left(\sum_{j=1}^{m}\left\langle\widehat{\mathrm{E}}\left(\lambda_{j}, d\right)-\widehat{\mathrm{E}}\left(\lambda_{j-1}, d\right) x, x\right\rangle\right)^{1 / 2}
\end{aligned}
$$




$$
\begin{aligned}
& \leq\left[2 \gamma_{1}+\gamma_{2}(d-c)\right]\left(\max _{j=1}\left|\delta_{j}^{(m)}\right|\right)^{1 / 2} \sqrt{b-a}(\langle\widehat{\mathrm{E}}(b, d)-\widehat{\mathrm{E}}(a, d) x, x\rangle)^{1 / 2} \\
& \leq\left[2 \gamma_{1}+\gamma_{2}(d-c)\right]\left(\max _{j=1}^{m}\left|\delta_{j}^{(m)}\right|\right)^{1 / 2} \sqrt{b-a}\|\widehat{\mathrm{E}}(b, d)-\widehat{\mathrm{E}}(a, d)\|\|x\| .
\end{aligned}
$$

It is proven analogously that

$$
\left\|\sum_{j=1}^{m} T_{j k}^{(2)} x\right\| \leq\left[2 \gamma_{1}+\gamma_{2}(b-a)\right]\left|\omega_{k}^{(n)}\right|\left\|\left(\widehat{\mathrm{E}}\left(b, \mu_{k}\right)-\widehat{\mathrm{E}}\left(b, \mu_{k-1}\right)\right) x\right\|
$$

and then

$$
\left\|\sum_{j=1}^{m} \sum_{k=1}^{n} T_{j k}^{(2)} x\right\| \leq\left[2 \gamma_{1}+\gamma_{2}(b-a)\right]\left(\max _{k=1}^{n}\left|\omega_{k}^{(n)}\right|\right)^{1 / 2} \sqrt{d-c}\|\widehat{\mathrm{E}}(b, d)-\widehat{\mathrm{E}}(b, c)\|\|x\| .
$$

It only remains to find an estimate for the contribution to the difference 2.32 from the terms $T_{j k}^{(3)}$ given by 2.33). By using identity 2.5) and taking into account 2.13) it follows from $(2.33$ that

$$
\begin{aligned}
\left\|T_{j k}^{(3)} x\right\| & \leq \gamma_{2} \sum_{s=1}^{p_{j}-1} \sum_{t=1}^{q_{k}-1}\left|\lambda_{j, s}-\lambda_{j, s-1}\right|\left|\mu_{k, t}-\mu_{k, t-1}\right|\left\|\mathrm{E}\left(\left[\lambda_{j, s}, \lambda_{j}\right) \times\left[\mu_{k, t}, \mu_{k}\right)\right) x\right\| \\
& \leq \gamma_{2} \sum_{s=1}^{p_{j}-1} \sum_{t=1}^{q_{k}-1}\left|\lambda_{j, s}-\lambda_{j, s-1}\right|\left|\mu_{k, t}-\mu_{k, t-1}\right|\left\|\mathrm{E}\left(\delta_{j}^{(m)} \times \omega_{k}^{(n)}\right) x\right\| \\
& \leq \gamma_{2}\left|\lambda_{j, p_{j}-1}-\lambda_{j-1}\right|\left|\mu_{k, q_{k}-1}-\mu_{k-1}\right|\left\|\mathrm{E}\left(\delta_{j}^{(m)} \times \omega_{k}^{(n)}\right) x\right\| \\
& \leq \gamma_{2}\left|\delta_{j}^{(m)}\right|\left|\omega_{k}^{(n)}\right|\left\|\mathrm{E}\left(\delta_{j}^{(m)} \times \omega_{k}^{(n)}\right) x\right\|=\gamma_{2}\left|\delta_{j}^{(m)}\right|\left|\omega_{k}^{(n)}\right|\left\|\mathrm{E}\left(\Delta_{j k}^{(m, n)}\right) x\right\| .
\end{aligned}
$$

Hence

$$
\begin{aligned}
\left\|\sum_{j=1}^{m} \sum_{k=1}^{n} T_{j k}^{(3)} x\right\| & \leq \sum_{j=1}^{m} \sum_{k=1}^{n}\left\|T_{j k}^{(3)} x\right\| \\
\leq & \gamma_{2}\left(\sum_{j=1}^{m} \sum_{k=1}^{n}\left|\delta_{j}^{(m)}\right|^{2}\left|\omega_{k}^{(n)}\right|^{2}\right)^{1 / 2}\left(\sum_{j=1}^{m} \sum_{k=1}^{n}\left\|\mathrm{E}\left(\Delta_{j k}^{(m, n)}\right) x\right\|^{2}\right)^{1 / 2} \\
& =\gamma_{2}\left(\sum_{j=1}^{m}\left|\delta_{j}^{(m)}\right|^{2}\right)^{1 / 2}\left(\sum_{k=1}^{n}\left|\omega_{k}^{(n)}\right|^{2}\right)^{1 / 2}\left(\sum_{j=1}^{m} \sum_{k=1}^{n}\left\langle\mathrm{E}\left(\Delta_{j k}^{(m, n)}\right) x, x\right\rangle\right)^{1 / 2} \\
\leq & \gamma_{2}\left(\max _{j=1}^{m a x}\left|\delta_{j}^{(m)}\right| \max _{k=1}^{n}\left|\omega_{k}^{(n)}\right|\right)^{1 / 2} \sqrt{(b-a)(d-c)}\langle\mathrm{E}(\Delta) x, x\rangle^{1 / 2} \\
\leq & \gamma_{2}\left(\max _{j=1}^{m a x}\left|\delta_{j}^{(m)}\right| \max _{k=1}^{n}\left|\omega_{k}^{(n)}\right|\right)^{1 / 2} \sqrt{(b-a)(d-c)}\|\mathrm{E}(\Delta)\|\|x\|
\end{aligned}
$$

Now combining 2.32, 2.37, 2.38), and 2.40) one concludes that the integral sum 2.28 converges as $m \rightarrow \infty, n \rightarrow \infty, \max _{j=1}^{m}\left|\delta_{j}^{m}\right| \rightarrow 0$, and $\max _{k=1}^{n}\left|\omega_{k}^{n}\right| \rightarrow 0$ to a linear operator of $\mathscr{B}(\mathfrak{K}, \mathfrak{H})$. The convergence takes place with respect to the uniform operator topology.

The proof is complete. 
Remark 2.6. It is an open problem whether or not the sufficient conditions 2.12, 2.13) for a function $F: \Delta \rightarrow \mathscr{B}(\mathfrak{H}, \mathfrak{K})$ to be right-integrable on $\Delta$ with respect to the spectral measure $d E(\lambda, \mu)$ in the sense of the uniform operator topology are optimal. We also notice that even in the case where the spectral family $\{E(\cdot)\}$ is associated with a selfadjoint operator, a similar problem remains unsolved. More precisely, it is known that if an operator-valued function is Lipschitz on a finite interval in $\mathbb{R}$ then it is integrable on this interval in the sense of the uniform operator topology with respect to the spectral measure of any self-adjoint operator (see [1] Lemma 7.2 and Remark 7.3]). But, to the best of our knowledge, it is still unknown whether the requirement for the function to be Lipschitz is optimal.

Corollary 2.7. Assume that for some $\gamma_{1}, \gamma_{2}>0$ an operator-valued function $G: \Delta \rightarrow$ $\mathscr{B}(\mathfrak{H}, \mathfrak{K})$ satisfies conditions $(2.12)$ and $(2.13)$. Then $G$ is left-integrable on $\Delta$ with respect to the spectral measure $d \mathrm{E}(\lambda, \mu)$ in the sense of the uniform operator topology.

Proof. The statement is an immediate consequence of Theorem 2.5 by applying Lemma 2.3

Remark 2.8. If the integral (2.10) exists, its range (as that of an operator of $\mathscr{B}(\mathfrak{H}, \mathfrak{K})$ ) lies in $\mathfrak{K}_{\Delta}=\operatorname{Ran}(\mathrm{E}(\Delta))$.

The next lemma gives a norm estimate for the integral of an operator-valued function with respect to the spectral measure of a normal operator.

Lemma 2.9. Under the hypothesis of Theorem 2.5] the integral (2.9) satisfies the following norm estimate

$$
\begin{aligned}
\int_{\Delta} F(\lambda, \mu) d \mathrm{E}(\lambda, \mu) \leq & 4 \sup _{(\lambda, \mu) \in \Delta}\|F(\lambda, \mu)\| \\
& \left.+2 \gamma_{1}(b-a+d-c)+\gamma_{2}(b-a)(d-c)\right] .
\end{aligned}
$$

Proof. Assume that $a=\lambda_{0}<\lambda_{1}<\ldots<\lambda_{m}=b$ and $c=\mu_{0}<\mu_{1}<\ldots<\mu_{n}=b$. Let $\delta_{j}^{(m)}=\left[\lambda_{j-1}, \lambda_{j}\right), j=1,2, \ldots, m$, and $\omega_{k}^{(n)}=\left[\mu_{k-1}, \mu_{k}\right), k=1,2, \ldots, n$, be the corresponding partitions of the intervals $[a, b)$ and $[c, d)$. Also let $\Delta_{j k}^{(m, n)}=\delta_{k}^{(m)} \times \omega_{k}^{(n)}$. Observe that the integral sum

$$
J_{m, n}=\sum_{j=1}^{m} \sum_{k=1}^{n} F\left(\lambda_{j-1}, \mu_{k-1}\right) \mathrm{E}\left(\Delta_{j k}^{(m, n)}\right)
$$

reads

$$
J_{m, n}=\sum_{j=1}^{m} \sum_{k=1}^{n} F\left(\lambda_{j-1}, \mu_{k-1}\right)\left[\widehat{\mathrm{E}}\left(\lambda_{j}, \mu_{k}\right)-\widehat{\mathrm{E}}\left(\lambda_{j-1}, \mu_{k}\right)-\widehat{\mathrm{E}}\left(\lambda_{j}, \mu_{k-1}\right)+\widehat{\mathrm{E}}\left(\lambda_{j-1}, \mu_{k-1}\right)\right] .
$$

By regrouping the terms and taking into account that $\lambda_{0}=a, \lambda_{m}=b, \mu_{0}=c$, and $\mu_{n}=d$ one then verifies that

$$
J_{m, n}=S_{1}+S_{2}+S_{3}+S_{4},
$$

where

$$
\begin{aligned}
& S_{1}=F\left(\lambda_{m-1}, \mu_{n-1}\right) \widehat{\mathrm{E}}(b, d)-F\left(a, \mu_{n-1}\right) \widehat{\mathrm{E}}(a, d)-F\left(\lambda_{m-1}, c\right) \widehat{\mathrm{E}}(b, c)+F(a, c) \widehat{\mathrm{E}}(a, c), \\
& S_{2}=\sum_{j=1}^{m-1}\left\{\left[F\left(\lambda_{j-1}, \mu_{n-1}\right)-F\left(\lambda_{j}, \mu_{n-1}\right)\right] \widehat{\mathrm{E}}\left(\lambda_{j}, d\right)-\left[F\left(\lambda_{j-1}, c\right)-F\left(\lambda_{j}, c\right)\right] \widehat{\mathrm{E}}\left(\lambda_{j}, c\right)\right\},
\end{aligned}
$$




$$
S_{3}=\sum_{k=1}^{n-1}\left\{\left[F\left(\lambda_{m-1}, \mu_{k-1}\right)-F\left(\lambda_{m-1}, \mu_{k}\right)\right] \widehat{\mathrm{E}}\left(b, \mu_{k}\right)-\left[F\left(a, \mu_{k-1}\right)-F\left(a, \mu_{k}\right)\right] \widehat{\mathrm{E}}\left(a, \mu_{k}\right)\right\},
$$

and

$$
S_{4}=\sum_{j=1}^{m-1} \sum_{k=1}^{n-1}\left[F\left(\lambda_{j}, \mu_{k}\right)-F\left(\lambda_{j-1}, \mu_{k}\right)-F\left(\lambda_{j}, \mu_{k-1}\right)+F\left(\lambda_{j-1}, \mu_{k-1}\right)\right] \widehat{\mathrm{E}}\left(\lambda_{j}, \mu_{k}\right)
$$

We have

$$
\|\widehat{\mathrm{E}}(\lambda, \mu)\| \leq 1 \quad \text { for any } \quad \lambda, \mu \in \mathbb{R}
$$

and hence

$$
S_{1} \leq 4 \sup _{(\lambda, \mu) \in \Delta}\|F(\lambda, \mu)\| .
$$

By hypothesis the operator-valued function $F$ satisfies estimates (2.12) and 2.13). Taking into account (2.44), this implies

$$
\begin{aligned}
& S_{2} \leq 2 \gamma_{1} \sum_{j=1}^{m-1}\left|\lambda_{j-1}-\lambda_{j}\right|<2 \gamma_{1}(b-a), \\
& S_{3} \leq 2 \gamma_{1} \sum_{k=1}^{n-1}\left|\mu_{k-1}-\mu_{k}\right|<2 \gamma_{1}(d-c),
\end{aligned}
$$

and

$$
S_{4} \leq \gamma_{2} \sum_{j=1}^{m-1} \sum_{k=1}^{n-1}\left|\lambda_{j-1}-\lambda_{j}\right|\left|\mu_{k-1}-\mu_{k}\right|<\gamma_{2}(b-a)(d-c) .
$$

Combining (2.43 and 2.45-2.48 and passing in 2.42) to the limit as $\max _{j=1}^{m}\left|\delta_{j}^{(m)}\right|+$ $\max _{k=1}^{n}\left|\omega_{k}^{(n)}\right| \rightarrow 0$ one arrives at inequality (2.41) which completes the proof.

Now let $\Omega=\{z \in \mathbb{C} \mid a \leq \operatorname{Re} z<b, c \leq \operatorname{Im} z<d\}$ be a rectangle in $\mathbb{C}$ with finite $a, b, c$, and $d$. Assume that $F: \Omega \rightarrow \mathscr{B}(\mathfrak{K}, \mathfrak{H})$ and $G: \Omega \rightarrow \mathscr{B}(\mathfrak{H}, \mathfrak{K})$ are such that the corresponding functions $F(\lambda+\mathrm{i} \mu)$ and $G(\lambda+\mathrm{i} \mu)$ of real variables $\lambda \in[a, b)$ and $\mu \in[c, d)$ are resp. rightand left-integrable with the spectral measure $d \mathrm{E}(\lambda, \mu)$ over the rectangle $\Delta=[a, b) \times[c, d)$ in $\mathbb{R}^{2}$. In this case we set

$$
\begin{aligned}
& \int_{\Omega} F(z) d E(z)=\int_{\Delta} F(\lambda+\mathrm{i} \mu) d \mathrm{E}(\lambda, \mu), \\
& \int_{\Omega} d E(z) G(z)=\int_{\Delta} d \mathrm{E}(\lambda, \mu) G(\lambda+\mathrm{i} \mu),
\end{aligned}
$$

where $d E(z)$ stands for the spectral measure of the same normal operator as the measure $d \mathrm{E}(\lambda, \mu)$ but on the complex plane.

Let $F: \Omega \rightarrow \mathscr{B}(\mathfrak{K}, \mathfrak{H})$ and $G: \Omega \rightarrow \mathscr{B}(\mathfrak{H}, \mathfrak{K})$ are such that the $F(\lambda+\mathrm{i} \mu)$ and $G(\lambda+\mathrm{i} \mu)$, $(\lambda, \mu) \in \Delta$, satisfy on $\Delta$ conditions (2.12) and (2.13). Then the integrals 2.49) and (2.50) exist by Theorem 2.5 and Corollary 2.7 respectively. Clearly, by (2.9) and 2.10) only the values of $F$ and $G$ on the support of the measure $d \mathrm{E}(\lambda, \mu)$ contribute to these integrals. Assuming that $d E(z)$ (or, equivalently, $d \mathrm{E}(\lambda, \mu)$ ) is the spectral measure associated with a normal operator $C$, by

$$
\int_{\operatorname{spec}(C) \cap \Omega} F(z) d E(z)=\int_{\Omega} F(z) d E(z),
$$




$$
\int_{\operatorname{spec}(C) \cap \Omega} d E(z) G(z)=\int_{\Omega} d E(z) G(z)
$$

we define the corresponding integrals of $F$ and $G$ over the part of the spectrum of $C$ lying in $\Omega$.

In particular, if a function $F: \operatorname{spec}(C) \rightarrow \mathscr{B}(\mathfrak{K}, \mathfrak{H})($ resp. $G: \operatorname{spec}(C) \rightarrow \mathscr{B}(\mathfrak{H}, \mathfrak{K})$ ) defined (only) on the spectrum of $C$ admits an extension $\widetilde{F}$ (resp. $\widetilde{G}$ ) to the whole rectangle $\Omega$ in such a way that conditions (2.12) and (2.13) hold for $\widetilde{F}(\lambda+\mathrm{i} \mu)($ resp. for $\widetilde{G}(\lambda+\mathrm{i} \mu)$ ), $(\lambda, \mu) \in \Delta$, we set

$$
\begin{aligned}
\int_{\operatorname{spec}(C) \cap \Omega} F(z) d E(z) & =\int_{\Omega} \widetilde{F}(z) d E(z), \\
\int_{\operatorname{spec}(C) \cap \Omega} d E(z) G(z) & =\int_{\Omega} d E(z) \widetilde{G}(z) .
\end{aligned}
$$

Clearly, the results in 2.53) and 2.54) do not depend on the choice of the extensions $\widetilde{F}(z)$ and $\widetilde{G}(z)$ (provided that $\widetilde{F}(\lambda+\mathrm{i} \mu)$ and $\widetilde{G}(\lambda+\mathrm{i} \mu)$ satisfy (2.12) and (2.13).

Finally, the improper weak, strong, or uniform integrals

$$
\int_{a}^{b} \int_{c}^{d} F(\lambda, \mu) d \mathrm{E}(\lambda, \mu) \text { and } \int_{a}^{b} \int_{c}^{d} d \mathrm{E}(\lambda, \mu) G(\lambda, \mu)
$$

with infinite lower and/or upper bounds $(a=-\infty$ and/or $b=+\infty$ and $c=-\infty$ and/or $d=$ $+\infty)$ are understood as the limits, if they exist, of the integrals over finite intervals in the corresponding topologies. For example,

$$
\int_{-\infty}^{\infty} \int_{-\infty}^{\infty} d \mathrm{E}(\lambda, \mu) G(\lambda, \mu)=\lim _{\substack{a \downarrow-\infty, b \uparrow \infty \\ c \downarrow-\infty, d \uparrow \infty}} \int_{a}^{b} \int_{c}^{d} d \mathrm{E}(\lambda, \mu) G(\lambda, \mu) .
$$

If $d E(z)$ (or, equivalently, $d \mathrm{E}(\lambda, \mu)$ ) is the spectral measure associated with a normal operator $C$, we set

$$
\begin{aligned}
\int_{\operatorname{spec}(C)} F(z) d E(z) & =\int_{-\infty}^{+\infty} \int_{-\infty}^{+\infty} \widetilde{F}(\lambda+\mathrm{i} \mu) d \mathrm{E}(\lambda, \mu), \\
\int_{\operatorname{spec}(C)} d E(z) G(z) & =\int_{-\infty}^{+\infty} \int_{-\infty}^{+\infty} d \mathrm{E}(\lambda, \mu) \widetilde{G}(\lambda+\mathrm{i} \mu),
\end{aligned}
$$

assuming that $F: \operatorname{spec}(C) \rightarrow \mathscr{B}(\mathfrak{K}, \mathfrak{H})$ and $G: \operatorname{spec}(C) \rightarrow \mathscr{B}(\mathfrak{H}, \mathfrak{K})$ admit extensions $\widetilde{F}$ and $\widetilde{G}$ from $\operatorname{spec}(C)$ to the whole complex plane $\mathbb{C}^{2}$ in such a way that $\widetilde{F}(\lambda+\mathrm{i} \mu)$ and $\widetilde{G}(\lambda+\mathrm{i} \mu)$ satisfy conditions (2.12), 2.13) as functions of the variables $\lambda, \mu \in \mathbb{R}$.

We conclude this section with the the following natural result.

Lemma 2.10. Let $\mathfrak{H}$ and $\mathfrak{K}$ be Hilbert spaces. Assume that $C$ is a (possibly unbounded) normal operator on $\mathfrak{K}$ and $d E(z)$ is the spectral measure associated with $C$. Let $\Omega=\{z \in$ $\mathbb{C} \mid a \leq \operatorname{Re} z<b, c \leq \operatorname{Im} z<d\}$ with finite $a, b, c$, and $d$. Assume in addition that $G(z)$ is a $\mathscr{B}(\mathfrak{H}, \mathfrak{K})$-valued function holomorphic on an open circle in $\mathbb{C}$ containing the closure $\bar{\Omega}$ of 
the rectangle $\Omega$. Then

$$
\operatorname{Ran}\left(\int_{\Omega} d E(z) G(z)\right) \subset \operatorname{Dom}(C)
$$

and

$$
\int_{\Omega} d E(z)(z G(z))=C \int_{\Omega} d E(z) G(z) .
$$

Proof. Inclusion (2.56) is proven by Remark 2.8 taking into account definition 2.50) of the right-side integral of an operator-valued function with the measure $d E(z)$.

By hypothesis the functions $G(z)$ and $z G(z)$ are analytic on $\Omega$. Hence, written in terms of the variables $\lambda=\operatorname{Re} z \in[a, b), \mu=\operatorname{Im} z \in[c, d)$, they automatically satisfy conditions (2.12) and 2.13). Then by Theorem (2.5) both integrals

$$
\int_{\Omega} d E(z) G(z) \text { and } \int_{\Omega} d E(z)(z G(z))
$$

exist in the sense of the uniform operator topology.

Assume that the open circle mentioned in hypothesis is centered at the point $z_{0}$ and its radius equals $r$. Denote this circle by $C_{r}\left(z_{0}\right)$ and write the Taylor formula

$$
G(z)=\sum_{k=0}^{n} G_{k}\left(z-z_{0}\right)^{k}+R_{n}(z), \quad z \in C_{r}\left(z_{0}\right),
$$

where $G_{k}=\frac{G^{(k)}\left(z_{0}\right)}{k !}$ and $R_{n}(z)$ is the remainder term. Since the function $G(z)$ is holomorphic on $C_{r}\left(z_{0}\right)$ and the set $\Omega$ is a compact contained in $C_{r}\left(z_{0}\right)$, the remainder $R_{n}(z)$ and its derivatives converge to zero as $n \rightarrow \infty$ and the convergence is uniform with respect to $z \in \Omega$. In particular, this implies that

$$
\begin{gathered}
\sup _{\lambda+\mathrm{i} \mu \in \Omega}\left|S_{n}(\lambda, \mu)\right| \underset{n \rightarrow \infty}{\longrightarrow} 0, \\
\sup _{\lambda+\mathrm{i} \mu \in \Omega} \max \left\{\left|\frac{\partial S_{n}(\lambda, \mu)}{\partial \lambda}\right|,\left|\frac{\partial S_{n}(\lambda, \mu)}{\partial \mu}\right|\right\} \underset{n \rightarrow \infty}{\longrightarrow} 0,
\end{gathered}
$$

and

$$
\sup _{\lambda+\mathrm{i} \mu \in \Omega} \max \left\{\left|\frac{\partial^{2} S_{n}(\lambda, \mu)}{\partial \lambda^{2}}\right|,\left|\frac{\partial^{2} S_{n}(\lambda, \mu)}{\partial \mu^{2}}\right|,\left|\frac{\partial^{2} S_{n}(\lambda, \mu)}{\partial \lambda \partial \mu}\right|\right\} \underset{n \rightarrow \infty}{\longrightarrow} 0
$$

where

$$
S_{n}(\lambda, \mu)=z R_{n}(z)=(\lambda+\mathrm{i} \mu) R_{n}(\lambda+\mathrm{i} \mu), \quad \lambda \in[a, b), \mu \in[c, d) .
$$

From (2.60) and 2.61) it follows that there is sequence of $\gamma_{n}>0, n=0,1,2, \ldots$, such that

$$
\gamma_{n} \rightarrow 0 \text { as } n \rightarrow \infty
$$

and

$$
\begin{gathered}
\left\|S_{n}(\lambda, \mu)-S_{n}\left(\lambda^{\prime}, \mu^{\prime}\right)\right\| \leq \gamma_{n}\left(\left|\lambda-\lambda^{\prime}\right|+\left|\mu-\mu^{\prime}\right|\right), \\
\left\|S_{n}(\lambda, \mu)-S_{n}\left(\lambda^{\prime}, \mu\right)-S_{n}\left(\lambda, \mu^{\prime}\right)+S_{n}\left(\lambda^{\prime}, \mu^{\prime}\right)\right\| \leq \gamma_{n}\left|\lambda-\lambda^{\prime}\right|\left|\mu-\mu^{\prime}\right| \\
\text { for any } \lambda, \lambda^{\prime} \in[a, b) \text { and } \mu, \mu^{\prime} \in[c, d) .
\end{gathered}
$$

By combining Corollary 2.7 and Lemma 2.9 it follows from (2.59, (2.62) and (2.63) that

$$
\left\|\int_{\Omega} d E(z) z R_{n}(z)\right\| \underset{n \rightarrow \infty}{\longrightarrow} 0 .
$$


By a similar reasoning one also infers that

$$
\left\|\int_{\Omega} d E(z) R_{n}(z)\right\| \underset{n \rightarrow \infty}{\longrightarrow} 0
$$

and hence

$$
\left\|C \int_{\Omega} d E(z) R_{n}(z)\right\|=\left\|(C E(\Omega)) \int_{\Omega} d E(z) R_{n}(z)\right\| \underset{n \rightarrow \infty}{\longrightarrow} 0
$$

since the product $C E(\Omega)$ is a bounded operator.

By (2.58) we have

$$
\int_{\Omega} d E(z)\left(z G(z)=\sum_{k=0}^{n}\left(\int_{\Omega} z\left(z-z_{0}\right)^{k} d E(z)\right) G_{k}+\int_{\Omega} d E(z) z R_{n}(z)\right.
$$

which implies

$$
\begin{aligned}
\int_{\Omega} d E(z)(z G(z) & =\sum_{k=0}^{n} C\left(C-z_{0}\right)^{k} E(\Omega) G_{k}+\int_{\Omega} d E(z) z R_{n}(z) \\
& =C \sum_{k=0}^{n}\left(\int_{\Omega}\left(z-z_{0}\right)^{k} d E(z)\right) G_{k}+\int_{\Omega} d E(z) z R_{n}(z),
\end{aligned}
$$

by taking into account that $\operatorname{Ran} E(\Omega) \subset \operatorname{Dom}\left(C^{l}\right)$ for any $l=1,2, \ldots$ On the other hand by (2.58) it follows that

$$
C \int_{\Omega} d E(z) G(z)=C \sum_{k=0}^{n}\left(\int_{\Omega}\left(z-z_{0}\right)^{k} d E(z)\right) G_{k}+C \int_{\Omega} d E(z) R_{n}(z) .
$$

Comparing this equality with 2.66 yields

$$
\int_{\Omega} d E(z)\left(z G(z)-C \int_{\Omega} d E(z) G(z)=\int_{\Omega} d E(z) z R_{n}(z)-C \int_{\Omega} d E(z) R_{n}(z),\right.
$$

which by (2.64) and (2.65) completes the proof.

\section{NORM OF AN OPERATOR WITH RESPECT TO THE SPECTRAL MEASURE}

The paper [22] introduced the concept of the norm of a bounded operator with respect the spectral measure of a self-adjoint operator. This concept turned out to be a useful tool in the study of the operator Sylvester and Riccati equations (see [4] for details). The goal of the present section is to extend the above concept to the case where the spectral measure is associated with a normal operator.

Definition 3.1. Let $Y \in \mathscr{B}(\mathfrak{H}, \mathfrak{K})$ be a bounded operator from a Hilbert space $\mathfrak{H}$ to a Hilbert space $\mathfrak{K}$ and let $\{E(\Omega)\}_{\Omega \in \mathscr{A}_{\text {Borel }}(\mathbb{C})}$ be the spectral family of a (possibly unbounded) normal operator on $\mathfrak{K}$. Introduce

$$
\|Y\|_{E}=\left(\sup _{\left\{\Omega_{k}\right\}} \sum_{k}\left\|Y^{*} E\left(\Omega_{k}\right) Y\right\|\right)^{1 / 2},
$$

where the supremum is taken over finite (or countable) systems of mutually disjoint Borel subsets $\Omega_{k}$ of the complex plane $\mathbb{C}, \Omega_{k} \cap \Omega_{l}=\varnothing$, if $k \neq l$. The number $\|Y\|_{E}$ is called the norm of the operator $Y$ with respect to the spectral measure $d E(z)$ or simply $E$-norm of $Y$. For $Z \in \mathscr{B}(\mathfrak{K}, \mathfrak{H})$ the $E$-norm $\|Z\|_{E}$ is defined by $\|Z\|_{E}=\left\|Z^{*}\right\|_{E}$. 
One easily verifies that if the norm $\|Y\|_{E}$ is finite then

$$
\|Y\| \leq\|Y\|_{E} .
$$

If, in addition, $Y$ is a Hilbert-Schmidt operator, then

$$
\|Y\|_{E} \leq\|Y\|_{2}, \quad Y \in \mathscr{B}_{2}(\mathfrak{H}, \mathfrak{K}),
$$

where $\|\cdot\|_{2}$ denotes the (Hilbert-Schmidt) norm on the ideal $\mathscr{B}_{2}(\mathfrak{H}, \mathfrak{K})$ of Hilbert-Schmidt operators from $\mathfrak{H}$ to $\mathfrak{K}$.

The following statement is an extension of [22 Lemma 10.7].

Lemma 3.2. Assume that $C$ is a normal operator on the Hilbert space $\mathfrak{K}$. Let an operatorvalued function $F: \operatorname{spec}(C) \rightarrow \mathscr{B}(\mathfrak{H})$ be bounded

$$
\|F\|_{\infty}=\sup _{z \in \operatorname{spec}(C)}\|F(z)\|<\infty
$$

and admit a bounded extension as a function of $\lambda=\operatorname{Re} z$ and $\mu=\operatorname{Im} z$ from $\sigma(C)$ to the whole plane $\mathbb{R}^{2}$ which satisfies conditions (2.12) and 2.13. If the E-norm $\|Y\|_{E}$ of an operator $Y \in \mathscr{B}(\mathfrak{H}, \mathfrak{K})$ with respect to the spectral measure $d E(z)$ on $\mathbb{C}$ associated with $C$ is finite, then the integrals

$$
\int_{\operatorname{spec}(C)} d E(z) Y F(z) \text { and } \int_{\operatorname{spec}(C)} F(z) Y^{*} d E(z)
$$

exist in the uniform operator topology. Moreover, the following bounds hold

$$
\begin{aligned}
& \left\|\int_{\operatorname{spec}(C)} d E(z) Y F(z)\right\| \leq\|Y\|_{E} \cdot\|F\|_{\infty}, \\
& \left\|\int_{\operatorname{spec}(C)} F(z) Y^{*} d E(z)\right\| \leq\|Y\|_{E} \cdot\|F\|_{\infty} .
\end{aligned}
$$

Proof. The proof is given for the case of the integral in (3.3).

For the extension of the function $F(\lambda+\mathrm{i} \mu)$ from the set $\sigma(C)$ to $\mathbb{R}^{2}$ we will use the notation $F(\lambda, \mu), \lambda, \mu \in \mathbb{R}$.

Let $[a, b) \subset \mathbb{R}$ and $[c, d) \subset \mathbb{R}$ be finite intervals, $-\infty<a<b<\infty,-\infty<c<d<\infty$, and let $\left\{\delta_{j}^{(m)}\right\}_{j=1}^{m}$ and $\left\{\omega_{k}^{(n)}\right\}_{k=1}^{n}$ be partitions of $[a, b)$ and $[c, d)$, respectively. If $\left(\delta_{j}^{(m)} \times\right.$ $\left.\omega_{k}^{(n)}\right) \cap \sigma(C) \neq \varnothing$ choose $\xi_{j} \in \delta_{j}^{(m)}$ and $\zeta_{k} \in \omega_{k}^{(n)}$ in such a way that $\xi_{j}+\mathrm{i} \zeta_{k} \in \operatorname{spec}(C)$, that is, $\left(\xi_{j}, \zeta_{k}\right) \in \sigma(C)$. If $\left(\delta_{j}^{(m)} \times \omega_{k}^{(n)}\right) \cap \sigma(C)=\varnothing$ let $\xi_{j}$ and $\zeta_{k}$ be arbitrary points of the intervals $\delta_{j}^{(m)}$ and $\omega_{k}^{(n)}$, respectively. Then taking into account that

$$
\mathrm{E}\left(\delta_{j}^{(m)} \times \omega_{k}^{(n)}\right) \mathrm{E}\left(\delta_{s}^{(m)} \times \omega_{t}^{(n)}\right)=0 \text { if } j \neq s \text { or } k \neq t,
$$

for any $x \in \mathfrak{H}$ one obtains

$$
\begin{aligned}
& \left\|\sum_{j=1}^{m} \sum_{k=1}^{n} \mathrm{E}\left(\delta_{j}^{(m)} \times \omega_{k}^{(n)}\right) Y F\left(\xi_{j}, \zeta_{k}\right) x\right\|^{2} \\
& \quad=\left\langle\sum_{j=1}^{m} \sum_{k=1}^{n} \mathrm{E}\left(\delta_{j}^{(m)} \times \omega_{k}^{(n)}\right) Y F\left(\xi_{j}, \zeta_{k}\right) x, \sum_{s=1}^{m} \sum_{t=1}^{n} \mathrm{E}\left(\delta_{s}^{(m)} \times \omega_{t}^{(n)}\right) Y F\left(\xi_{s}, \zeta_{t}\right) x\right\rangle
\end{aligned}
$$




$$
\begin{aligned}
& =\left\langle\sum_{j=1}^{m} \sum_{k=1}^{n}\left[F\left(\xi_{j}, \zeta_{k}\right)\right]^{*} Y^{*} \mathrm{E}\left(\delta_{j}^{(m)} \times \omega_{k}^{(n)}\right) Y F\left(\xi_{j}, \zeta_{k}\right) x, x\right\rangle \\
& =\left\langle\sum_{j, k:\left(\xi_{j}, \zeta_{k}\right) \in \sigma(C)}\left[F\left(\xi_{j}, \zeta_{k}\right)\right]^{*} Y^{*} \mathrm{E}\left(\delta_{j}^{(m)} \times \omega_{k}^{(n)}\right) Y F\left(\xi_{j}, \zeta_{k}\right) x, x\right\rangle .
\end{aligned}
$$

Hence

$$
\begin{aligned}
\| \sum_{j=1}^{m} \sum_{k=1}^{n} \mathrm{E} & \left(\delta_{j}^{(m)} \times \omega_{k}^{(n)}\right) Y F\left(\xi_{j}, \zeta_{k}\right) x \|^{2} \\
& \leq \sup _{(\xi, \zeta) \in \sigma(C)}\|F(\xi, \zeta)\|^{2} \sum_{j, k:\left(\xi_{j}, \zeta_{k}\right) \in \sigma(C) \cap \Delta}\left\|Y^{*} \mathrm{E}\left(\delta_{j}^{(m)} \times \omega_{k}^{(n)}\right) Y\right\|\|x\|^{2} \\
& \leq\|F\|_{\infty}^{2}\|Y\|_{E, \Delta}^{2}\|x\|^{2},
\end{aligned}
$$

where $\Delta=[a, b) \times[c, d)$ and

$$
\|Y\|_{E, \Delta}=\left(\sup _{\left\{\Delta_{k}\right\}} \sum_{k}\left\|Y^{*} \mathrm{E}\left(\Delta_{k}\right) Y\right\|\right)^{1 / 2} .
$$

In (3.6) the supremum is taken over finite (or countable) systems of Borel subsets $\Delta_{k}$ of the rectangle $\Delta$ such that $\Delta_{k} \cap \Delta_{l}=\varnothing$, if $k \neq l$. Obviously,

$$
\|Y\|_{E, \Delta} \leq\|Y\|_{E} .
$$

and

$$
\lim _{\substack{a \downarrow-\infty, b \uparrow \infty \\ c \downarrow-\infty, d \uparrow \infty}}\|Y\|_{E, \Delta}=\|Y\|_{E} .
$$

By hypothesis the function $F(\lambda, \mu)$ satisfies the assumptions of Theorem 2.5 and hence by Corollary 2.7 it is right-integrable on the rectangle $\Delta$ with respect to the measure $d \mathrm{E}(\lambda, \mu)$. From 3.5 it follows that

$$
\left\|\int_{\Delta} d \mathrm{E}(\lambda, \mu) Y F(\lambda, \mu)\right\| \leq\|F\|_{\infty}\|Y\|_{E, \Delta}
$$

Thus 3.8 implies that

$$
\left\|\int_{\left[a^{\prime}, b^{\prime}\right) \times\left[c^{\prime}, d^{\prime}\right)} d \mathrm{E}(\lambda, \mu) Y F(\lambda, \mu)\right\| \rightarrow 0
$$

as $a^{\prime} \rightarrow \infty$ or $b^{\prime} \rightarrow-\infty \quad\left(\right.$ for $\left.b^{\prime}>a^{\prime}\right)$ and/or $c^{\prime} \rightarrow \infty$ or $d^{\prime} \rightarrow-\infty \quad\left(\right.$ for $\left.d^{\prime}>c^{\prime}\right)$,

which together with 3.9 proves the existence of the limit

$$
\int_{\mathbb{R}^{2}} d \mathrm{E}(\lambda, \mu) Y F(\lambda, \mu)=\lim _{\substack{a \downarrow-\infty, b \uparrow \infty \\ c \downarrow-\infty, d \uparrow \infty}} \int_{[a, b) \times[c, d)} d \mathrm{E}(\lambda, \mu) Y F(\lambda, \mu)
$$

and hence the convergence of the integral $\int_{\operatorname{spec}(C)} d E(z) Y F(z)$ in the sense of the operator norm topology as well as the bound (3.3). Then the convergence of the integral $\int_{\operatorname{spec}(C)} F(z) Y^{*} d E(z)$ with respect to the operator norm topology and the norm estimate (3.4) are proven by applying Lemma 2.3 
The proof is complete.

\section{SylVESTER EQUATION}

Assuming that the entry $C$ in the operator Sylvester equation 1.8 is a normal operator, the principal goal of this section is to introduce a Stieltjes integral representation for the solution $X$ in terms of the spectral measure associated with $C$.

We begin with recalling the concepts of weak, strong, and operator solutions to the operator Sylvester equations.

Definition 4.1. Let $A$ and $C$ be densely defined possibly unbounded closed operators on Hilbert spaces $\mathfrak{H}$ and $\mathfrak{K}$, respectively. A bounded operator $X \in \mathscr{B}(\mathfrak{H}, \mathfrak{K})$ is said to be a weak solution of the Sylvester equation

$$
X A-C X=D, \quad D \in \mathscr{B}(\mathfrak{H}, \mathfrak{K}),
$$

if

$$
\langle X A f, g\rangle-\left\langle X f, C^{*} g\right\rangle=\langle D f, g\rangle \quad \text { for all } f \in \operatorname{Dom}(A) \text { and } g \in \operatorname{Dom}\left(C^{*}\right) .
$$

A bounded operator $X \in \mathscr{B}(\mathfrak{H}, \mathfrak{K})$ is said to be a strong solution of the Sylvester equation (4.1) if

$$
\operatorname{Ran}\left(\left.X\right|_{\operatorname{Dom}(A)}\right) \subset \operatorname{Dom}(C),
$$

and

$$
X A f-C X f=D f \quad \text { for all } f \in \operatorname{Dom}(A) .
$$

Finally, a bounded operator $X \in \mathscr{B}(\mathfrak{H}, \mathfrak{K})$ is said to be an operator solution of the Sylvester equation (4.1) if

$$
\operatorname{Ran}(X) \subset \operatorname{Dom}(C),
$$

the operator $X A$ is bounded on $\operatorname{Dom}(X A)=\operatorname{Dom}(A)$, and the equality

$$
\overline{X A}-C X=D
$$

holds as an operator equality, where $\overline{X A}$ denotes the closure of $X A$.

Along with the Sylvester equation 4.1 we also introduce the dual equation

$$
Y C^{*}-A^{*} Y=D^{*}
$$

for which the notion of weak, strong, and operator solutions is defined in a way analogous to that in Definition 4.1

Clearly, if $X \in \mathscr{B}(\mathfrak{K}, \mathfrak{H})$ is an operator solution of the Sylvester equation 4.1), it is also a strong solution to 4.1. In its turn, any strong solution is also a weak solution. In fact, one does not need to distinguish between weak and strong solutions to the Sylvester equation (4.1) since any weak solution to this equation is in fact a strong solution.

Lemma 4.2. Let $A$ and $C$ be densely defined possibly unbounded closed operators on the Hilbert spaces $\mathfrak{H}$ and $\mathfrak{K}$, respectively. If $X \in \mathscr{B}(\mathfrak{K}, \mathfrak{H})$ is a weak solution of the Sylvester equation (4.1) then $X$ is a strong solution of 4.1), too.

Proof. Given $f \in \operatorname{Dom}(A)$, introduce a linear functional $\mathfrak{l}_{f}$ with $\operatorname{Dom}\left(\mathfrak{l}_{f}\right)=\operatorname{Dom}\left(C^{*}\right)$ by

$$
\mathfrak{l}_{f}(g)=\left\langle C^{*} g, X f\right\rangle=\langle g, X A f\rangle-\langle g, D f\rangle, \quad g \in \operatorname{Dom}\left(C^{*}\right) .
$$

Clearly, for any $f \in \operatorname{Dom}(A)$ the functional $\mathfrak{l}_{f}$ is bounded,

$$
\left|\mathfrak{l}_{f}(g)\right|=\left|\left\langle C^{*} g, X f\right\rangle\right| \leq c_{f}\|g\|, \quad g \in \operatorname{Dom}\left(C^{*}\right),
$$


where $c_{f}=\|X A f\|+\|D f\|$. The functional $\mathfrak{l}_{f}$ is also densely defined since $\operatorname{Dom}\left(\mathfrak{l}_{f}\right)=$ $\operatorname{Dom}\left(C^{*}\right)$ is dense in $\mathfrak{K}$ as domain of adjoint of a closed densely defined operator. Thus, $X f \in \operatorname{Dom}\left(\left(C^{*}\right)^{*}\right)=\operatorname{Dom}(C)$ which implies that 4.3 holds and

$$
\langle g, X A f-C X f-D f\rangle=0 \text { for all } g \in \mathfrak{H} .
$$

Hence (4.4) also holds, completing the proof.

It is easy to see that if one of the equations 4.1) or (4.6) has a weak (and hence strong) solution then so does the other one.

Lemma 4.3. An operator $X \in \mathscr{B}(\mathfrak{H}, \mathfrak{K}$ ) is a weak (and hence strong) solution to the Sylvester equation (4.1) if and only if the operator $Y=-X^{*}$ is a weak (and hence strong) solution to the dual Sylvester equation (4.6.

Proof. The assertion is proven by combining Lemma 4.2] with [4] Lemma 2.4].

It is well known that if the spectra of the operators $A$ and $C$ are disjoint and one of them is a bounded operator then the Sylvester equation $X A-C X=D$ has a unique solution. This was first proven by by M. Krein in 1948. Later, the same result was independently obtained by Y. Daleckii [11] and M. Rosenblum [28]. The precise statement is as follows.

Lemma 4.4. Let A be a possibly unbounded densely defined closed operator in the Hilbert space $\mathfrak{H}$ and $C$ a bounded operator on the Hilbert space $\mathfrak{K}$ such that

$$
\operatorname{spec}(A) \cap \operatorname{spec}(C)=\varnothing
$$

and $D \in \mathscr{B}(\mathfrak{H}, \mathfrak{K})$. Then the Sylvester equation (4.1) has a unique operator solution

$$
X=\frac{1}{2 \pi \mathrm{i}} \int_{\Gamma} d \zeta(C-\zeta)^{-1} D(A-\zeta)^{-1},
$$

where $\Gamma$ is a union of closed contours in the complex plane with total winding number 0 around every point of $\operatorname{spec}(A)$ and total winding number 1 around every point of $\operatorname{spec}(C)$. Moreover,

$$
\|X\| \leq(2 \pi)^{-1}|\Gamma| \sup _{\zeta \in \Gamma}\left\|(C-\zeta)^{-1}\right\|\left\|(A-\zeta)^{-1}\right\|\|D\|,
$$

where $|\Gamma|$ denotes the length of the contour $\Gamma$.

A relatively recent review of results on the Sylvester operator equation 4.1 with both bounded entries $A$ and $C$ and applications of them to various problems can be found in [5].

If $A$ and $C$ are unbounded densely defined closed operators, even with separated spectra, then the Sylvester equation (4.1) may not have bounded solutions at all (a corresponding example can be found in [27|). Nevertheless, under some additional assumptions equation 4.1 is still solvable. A review of known sufficient conditions for solvability of 4.1 in the case where both $A$ and $C$ are allowed to be unbounded operators can be found in 4 Section 2].

Now we prove the main result of this section: if either $A$ or $C$ is normal, then a strong solution to the Sylvester equation, if it exists, can be represented in the form of an operator Stieltjes integral. The corresponding representation replaces the formula 4.77 where the contour integration might be impossible to perform in case of an unbounded operator $C$.

Theorem 4.5. Let A be a possibly unbounded densely defined closed operator on the Hilbert space $\mathfrak{H}$ and $C$ a normal operator on the Hilbert space $\mathfrak{K}$ with the spectral family

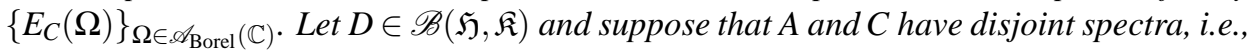

$$
\operatorname{dist}(\operatorname{spec}(A), \operatorname{spec}(C))>0 .
$$


Then the following statements are valid:

(i) Assume that the Sylvester equation (4.1) has a strong solution $X \in \mathscr{B}(\mathfrak{H}, \mathfrak{K})$. Then $X$ is a unique strong solution to (4.1) and it can be represented in the form of the Stieltjes integral

$$
X=\int_{\operatorname{spec}(C)} d E_{C}(\zeta) D(A-\zeta)^{-1},
$$

which converges in the sense of the strong operator topology in $\mathscr{B}(\mathfrak{H}, \mathfrak{K})$.

Conversely, if the Stieltjes integral (4.9) exists in the sense of the strong operator topology, then X given by (4.9) is a strong solution to (4.1).

(ii) Assume that the dual Sylvester equation

$$
Y C^{*}-A^{*} Y=D^{*}
$$

has a strong solution $Y \in \mathscr{B}(\mathfrak{K}, \mathfrak{H})$. Then $Y$ is a unique strong solution to (4.10) and it can be represented in the form of the Stieltjes operator integral

$$
Y=-\int_{\operatorname{spec}\left(C^{*}\right)}\left(A^{*}-\zeta\right)^{-1} D^{*} E_{C^{*}}(d \zeta)
$$

that exists in the sense of the strong operator topology in $\mathscr{B}(\mathfrak{K}, \mathfrak{H})$.

Proof. (i) Set $\Delta=[a, b) \times[c, d)$ where $[a, b)$ and $[c, d)$ are finite real intervals. Let $\left\{\delta_{j}\right\}$ be a finite system of mutually disjoint intervals such that $[a, b)=\cup_{j} \delta_{j}$ and $\left\{\omega_{k}\right\}$ another finite system of mutually disjoint intervals such that $[c, d)=\cup_{k} \omega_{k}$. Further, introduce the partition rectangles $\Delta_{j k}=\delta_{j} \times \omega_{k}$. For the pairs $j, k$ such that $\Delta_{j k} \cap \sigma(C) \neq \varnothing$ pick $\zeta_{\Delta_{j k}} \in \mathbb{C}$ such that the point $\left(\operatorname{Re} \zeta_{\Delta_{j k}}, \operatorname{Im} \zeta_{\Delta_{j k}}\right) \in \mathbb{R}^{2}$ belongs to the intersection $\Delta_{j k} \cap \sigma(C)$. Applying to both sides of (4.4) the spectral projection $\mathrm{E}_{C}\left(\Delta_{j k}\right)$, a short computation yields

$$
\mathrm{E}_{C}\left(\Delta_{j k}\right) X A f-\zeta_{\Delta_{j k}} \mathrm{E}_{C}\left(\Delta_{j k}\right) X f=\mathrm{E}_{C}\left(\Delta_{j k}\right) D f+\mathrm{E}_{C}\left(\Delta_{j k}\right)\left(C-\zeta_{\Delta_{j k}}\right) X f
$$

for any $f \in \operatorname{Dom}(A)$. Since $\left(\operatorname{Re} \zeta_{\Delta_{j k}}, \operatorname{Im} \zeta_{\Delta_{j k}}\right) \in \Delta_{j k} \cap \sigma(C)$, by (4.8) one concludes that $\zeta_{\Delta_{j k}}$ belongs to the resolvent set of the operator $A$. Hence, (4.12) implies

$$
\mathrm{E}_{C}\left(\Delta_{j k}\right) X=\mathrm{E}_{C}\left(\Delta_{j k}\right) D\left(A-\zeta_{\Delta_{j k}}\right)^{-1}+\left(C-\zeta_{\Delta_{j k}}\right) \mathrm{E}_{C}\left(\Delta_{j k}\right) X\left(A-\zeta_{\Delta_{j k}}\right)^{-1} .
$$

Using 4.13) one obtains

$$
\begin{aligned}
\sum_{j, k: \Delta_{j, k} \cap \sigma(C) \neq \varnothing} \mathrm{E}_{C}\left(\Delta_{j, k}\right) X= & \sum_{j, k: \Delta_{j, k} \cap \sigma(C) \neq \varnothing} \mathrm{E}_{C}\left(\Delta_{j, k}\right) D\left(A-\zeta_{\Delta_{j, k}}\right)^{-1} \\
& +\sum_{j, k: \Delta_{j k} \cap \sigma(C) \neq \varnothing}\left(C-\zeta_{\Delta_{j, k}}\right) \mathrm{E}_{C}\left(\Delta_{j, k}\right) X\left(A-\zeta_{\Delta_{j, k}}\right)^{-1} .
\end{aligned}
$$

The left hand side of 4.14 can be computed explicitly:

$$
\sum_{\Delta_{j k} \cap \sigma(C) \neq \varnothing} \mathrm{E}_{C}\left(\Delta_{j k}\right) X=\mathrm{E}_{C}(\Delta \cap \sigma(C)) X=\mathrm{E}_{C}(\Delta) X=E_{C}(\Omega) X,
$$

where

$$
\Omega=\{\zeta \in \mathbb{C} \mid a \leq \operatorname{Re} \zeta<b, c \leq \operatorname{Im} \zeta<d\}
$$

is the imbedding of the rectangle $\Delta$ into the complex plane $\mathbb{C}$. Below we will also write the set $\Omega$ in the form

$$
\Omega=[a, b) \times \mathrm{i}[c, d) .
$$


The first term on the right-hand side of (4.14) is the integral sum for the Stieltjes integral (4.9). More precisely, since $(A-\zeta)^{-1}$ is analytic in a complex neighborhood of $\Omega \cap$ $\operatorname{spec}(C)$, by Theorem 2.5 and definitions (2.52, 2.54) one infers

$$
\begin{gathered}
\max _{j, k} \underset{\left.j\left|\delta_{j}\right|+\left|\omega_{k}\right|\right) \rightarrow 0}{ } \sum_{j, k: \Delta_{j, k} \cap \sigma(C) \neq \varnothing} \mathrm{E}_{C}\left(\Delta_{j, k}\right) D\left(A-\zeta_{\Delta_{j, k}}\right)^{-1} \\
=\int_{\operatorname{spec}(C) \cap \Omega} d E_{C}(\zeta) D(A-\zeta)^{-1} .
\end{gathered}
$$

By the same reasoning for the last term on the right-hand side of (4.14 we have

$$
\begin{aligned}
& \max _{j, k}\left(\left|\delta_{j}\right|+\left|+\omega_{k}\right|\right) \rightarrow 0 \\
& \quad \sum_{j, k: \Delta_{j, k} \cap \sigma(C) \neq \varnothing}\left(C-\zeta_{\Delta_{j, k}}\right) \mathrm{E}_{C}\left(\Delta_{j, k}\right) X\left(A-\zeta_{\Delta_{j, k}}\right)^{-1} \\
& \quad C C \int_{\operatorname{spec}(C) \cap \Omega} d E_{C}(\zeta) X(A-\zeta)^{-1}-\int_{\operatorname{spec}(C) \cap \Omega} d E_{C}(\zeta) \zeta X(A-\zeta)^{-1} .
\end{aligned}
$$

By using Lemma 2.10 one easily proves that the right-hand side of 4.18 is zero, that is,

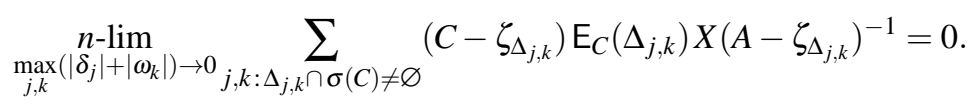

Passing to the limit $\max _{j, k}\left(\left|\delta_{j}\right|+\left|\omega_{k}\right|\right) \rightarrow 0$ in 4.14, by combining 4.15, 4.17, and (4.19) one concludes that for any finite rectangle $\Omega$ of the form (4.16)

$$
E_{C}(\Omega) X=\int_{\operatorname{spec}(C) \cap \Omega} d E_{C}(\zeta) D(A-\zeta)^{-1} .
$$

Since

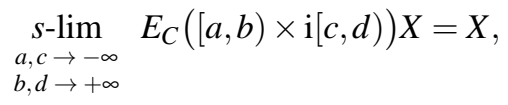

(4.20) implies (4.9), which, in particular, proves the uniqueness of a strong solution to the Riccati equation 4.1).

In order to prove the converse statement of $(i)$, assume that the Stieltjes integral on the right-hand side of 4.20) converges in the strong operator topology as $a, c \rightarrow-\infty$ and $b, d \rightarrow+\infty$ in 4.16 . Denote the resulting integral by $X$. Then, (4.20) holds for any finite rectangle $\Omega$ of the form 4.16 . This implies that for any $f \in \operatorname{Dom}(A)$ we have

$$
\begin{aligned}
& C E_{C}(\Omega) X f-E_{C}(\Omega) X A f \\
& \quad=\int_{\operatorname{spec}(C) \cap \Omega} d E_{C}(\zeta) D(A-\zeta)^{-1}(\zeta-A) f \\
& \quad=-\int_{\operatorname{spec}(C) \cap \Omega} d E_{C}(\zeta) D f=-E_{C}(\Omega) D f .
\end{aligned}
$$

Hence,

$$
\begin{aligned}
C E_{C}(\Omega) X f= & E_{C}(\Omega) X A f-E_{C}(\Omega) D f \\
= & E_{C}(\Omega)(X A f-D f) \\
& \quad \text { for any } f \in \operatorname{Dom}(A) .
\end{aligned}
$$


In particular, 4.21) implies that $C E_{C}(\Omega) X f$ with $\Omega$ given by (4.16) converges to $X A f-D f$ as $a, c \rightarrow-\infty$ and $b, d \rightarrow+\infty$. Since $\int_{\operatorname{spec}(C) \cap \Omega} \zeta d E_{C}(\zeta) X f=C E_{C}(\Omega) X f$, from (4.21) it also follows that

$$
\int_{\operatorname{spec}(C) \cap \Omega}|\zeta|^{2} d\left\langle E_{C}(\zeta) X f, X f\right\rangle=\sup _{\Omega \in \mathscr{P}}\left\|E_{C}(\Omega)(X A f-D f)\right\|^{2}=\|X A f-D f\|^{2}<\infty,
$$

where $\mathscr{P}$ stands for the set of all rectangles in $\mathbb{C}$ of the form 4.16. Hence,

$$
X f \in \operatorname{Dom}(C) \text {. }
$$

Then 4.21) can be rewritten as

$$
E_{C}(\Omega) C X f=E_{C}(\Omega)(X A f-D f) \text { for any } \Omega \in \mathscr{P} .
$$

Combining (4.22) and (4.23) proves that $X$ is a strong solution to the Sylvester equation 4.1.

(ii) Assume that the dual Sylvester equation (4.6) has a strong solution $Y \in \mathscr{B}(\mathfrak{K}, \mathfrak{H})$. As in the proof of $(i)$, choose a finite rectangle $\Delta \subset \mathbb{R}^{2}$ such that $\Delta \cap \sigma\left(C^{*}\right) \neq \varnothing$. Since $\mathrm{E}_{C^{*}}(\Delta) \mathfrak{K} \subset \operatorname{Dom}\left(C^{*}\right)$, we have $Y \mathrm{E}_{C^{*}}(\Delta) f \in \operatorname{Dom}\left(A^{*}\right)$ for any $f \in \mathfrak{K}$ by the definition of a strong solution. Take a point $\zeta_{\Delta} \in \operatorname{spec}\left(C^{*}\right)$ such that $\left(\operatorname{Re} \zeta_{\Delta}, \operatorname{Im} \zeta_{\Delta}\right) \in \Delta$. It follows from (4.8) that $\zeta_{\Delta} \notin \operatorname{spec}\left(A^{*}\right)$. As in the proof of $(i)$, it is easy to verify that

$$
Y \mathrm{E}_{C^{*}}(\Delta) f=-\left(A^{*}-\zeta_{\Delta}\right)^{-1} D^{*} \mathrm{E}_{C^{*}}(\Delta) f-\left(A^{*}-\zeta_{\Delta}\right)^{-1} Y\left(C^{*}-\zeta_{\Delta}\right) \mathrm{E}_{C^{*}}(\Delta) f,
$$

which holds for any $f \in \mathfrak{K}$.

Next, let $[a, b)$ be a finite interval and $\left\{\boldsymbol{\delta}_{j}\right\}$ a finite system of mutually disjoint intervals such that $[a, b)=\cup_{j} \delta_{j}$. Similarly, let $\left\{\omega_{k}\right\}$ be a finite system of mutually disjoint intervals partitioning a finite interval $[c, d)$, i.e., $\cup_{k} \omega_{k}=[c, d)$. Set $\Delta_{j, k}=\delta_{j} \times \omega_{k}$. For $j, k$ such that $\Delta_{j, k} \cap \sigma\left(C^{*}\right) \neq \varnothing$ pick a point $\zeta_{\Delta_{j, k}} \in \operatorname{spec}\left(C^{*}\right)$ such that $\left(\operatorname{Re} \zeta_{\Delta_{j, k}} \operatorname{Im} \zeta_{\Delta_{j, k}}\right) \in \Delta_{j, k}$. Using 4.24) one then finds that

$$
\begin{aligned}
Y \mathrm{E}_{C^{*}}([a, b) \times[c, d)) f= & -\sum_{j, k: \Delta_{j, k} \cap \operatorname{spec}\left(C^{*}\right) \neq \varnothing}\left(A^{*}-\zeta_{\Delta_{j, k}}\right)^{-1} D^{*} \mathrm{E}_{C^{*}}\left(\Delta_{j, k}\right) f \\
& -\sum_{j, k: \Delta_{j, k} \cap \operatorname{spec}\left(C^{*}\right) \neq \varnothing}\left(A^{*}-\zeta_{\Delta_{j, k}}\right)^{-1} Y\left(C^{*}-\zeta_{\Delta_{j, k}}\right) \mathrm{E}_{C^{*}}\left(\Delta_{j, k}\right) f .
\end{aligned}
$$

Equality 4.19) implies

$$
\sum_{j, k}^{n-\lim _{j}\left(\left|\delta_{j}\right|+\left|\omega_{k}\right|\right) \rightarrow 0} \sum_{j, k: \Delta_{j, k} \cap \operatorname{spec}\left(C^{*}\right) \neq \varnothing}\left(A^{*}-\zeta_{\Delta_{j, k}}\right)^{-1} Y\left(C^{*}-\zeta_{\Delta_{j, k}}\right) \mathrm{E}_{C^{*}}\left(\Delta_{j, k}\right)=0 .
$$

Thus, passing in 4.25) to the limit as $\max _{j, k}\left(\left|\delta_{j}\right|+\left|\omega_{k}\right|\right) \rightarrow 0$ one infers that

$$
-\int_{\operatorname{spec}\left(C^{*}\right) \cap \Omega}\left(A^{*}-\zeta\right)^{-1} D^{*} d E_{C^{*}}(\zeta) f=Y \mathrm{E}_{C^{*}}([a, b) \times[c, d)) f,
$$

where $\Omega$ is given by 4.16 . Since for any $f \in \mathfrak{K}$

$$
\lim _{\substack{a, c \rightarrow-\infty \\ b, d \rightarrow+\infty}} Y \mathrm{E}_{C^{*}}([a, b) \times[c, d)) f=Y,
$$

one concludes that the integral on the right-hand side of (4.27) converges as $a, c \rightarrow-\infty$ and $b, d \rightarrow+\infty$ in the strong operator topology and thus 4.11 holds, which gives a unique strong solution to the dual Sylvester equation (4.10). 
In order to prove the converse statement of $(i i)$, assume that there exists the strong operator limit

$$
Y=\operatorname{cim}_{\substack{a, c \rightarrow-\infty \\ b, d \rightarrow+\infty}} \int_{\operatorname{spec}\left(C^{*}\right) \cap([a, b) \times \mathrm{i}[c, d))}\left(A^{*}-\zeta\right)^{-1} D^{*} d E_{C^{*}}(\zeta), \quad Y \in \mathscr{B}(\mathfrak{K}, \mathfrak{H}) .
$$

Then for any finite $a, b, c$, and $d$ such that $a<b$ and $c<d$ we have

$$
Y E_{C^{*}}([a, b) \times \mathrm{i}[c, d))=-\int_{\operatorname{spec}\left(C^{*}\right) \cap([a, b) \times \mathrm{i}[c, d))}\left(A^{*}-\zeta\right)^{-1} D^{*} d E_{C^{*}}(\zeta) .
$$

By (4.8) any point $\xi \in \operatorname{spec}\left(C^{*}\right)$ belongs to the resolvent set of the operator $A$ and, hence, to the one of $A^{*}$. Picking such a $\xi \in \operatorname{spec}\left(C^{*}\right)$, the operator 4.29) can be split into two parts

$$
Y E_{C^{*}}([a, b) \times[c, d))=J_{1}(a, b, c, d)+J_{2}(a, b, c, d),
$$

where

$$
\begin{aligned}
& J_{1}(a, b, c, d)=-\left(A^{*}-\xi\right)^{-1} D^{*} E_{C^{*}}([a, b) \times \mathrm{i}[c, d)), \\
& J_{2}(a, b, c, d)=+\left(A^{*}-\xi\right)^{-1} \int_{\operatorname{spec}\left(C^{*}\right) \cap([a, b) \times \mathrm{i}[c, d))}(\xi-\zeta)\left(A^{*}-\zeta\right)^{-1} D^{*} d E_{C^{*}}(\zeta) .
\end{aligned}
$$

Using the functional calculus for the normal operator $C^{*}$ one obtains

$$
\begin{aligned}
J_{2}(a, b, c, d) f= & -\left(A^{*}-\xi\right)^{-1}\left(\int_{\operatorname{spec}\left(C^{*}\right) \cap([a, b) \times \mathrm{i}[c, d))}\left(A^{*}-\zeta\right)^{-1} D^{*} d E_{C^{*}}(\zeta)\right)\left(C^{*}-\xi\right) f \\
& \text { for any } f \in \operatorname{Dom}\left(C^{*}\right) .
\end{aligned}
$$

Thus, for $f \in \operatorname{Dom}\left(C^{*}\right)$ one concludes that

$$
\begin{aligned}
Y f= & \lim _{\substack{a, c \rightarrow-\infty \\
b, d \rightarrow+\infty}} Y E_{C^{*}}([a, b) \times \mathrm{i}[c, d)) f \\
= & \lim _{\substack{a, c \rightarrow-\infty \\
b, d \rightarrow+\infty}} J_{1}(a, b, c, d) f+\lim _{\substack{a, c \rightarrow-\infty \\
b, d \rightarrow+\infty}} J_{2}(a, b, c, d) f \\
= & -\left(A^{*}-\xi\right)^{-1} D^{*} f \\
& -\left(A^{*}-\xi\right)^{-1}\left(\int_{\operatorname{spec}\left(C^{*}\right)}\left(A^{*}-\zeta\right)^{-1} D^{*} d E_{C^{*}}(\zeta)\right)\left(C^{*}-\xi\right) f
\end{aligned}
$$

That is,

$$
Y f=-\left(A^{*}-\xi\right)^{-1} D^{*} f+\left(A^{*}-\xi\right)^{-1} Y\left(C^{*}-\xi\right) f, \quad f \in \operatorname{Dom}\left(C^{*}\right),
$$

since

$$
\begin{aligned}
& \int_{\operatorname{spec}\left(C^{*}\right)}\left(A^{*}-\zeta\right)^{-1} D^{*} d E_{C^{*}}(\zeta) \\
& =\operatorname{s-lim}_{\substack{s, c \rightarrow-\infty \\
b, d \rightarrow+\infty}} \int_{\operatorname{spec}\left(C^{*}\right) \cap([a, b) \times \mathrm{i}[c, d))}\left(A^{*}-\zeta\right)^{-1} D^{*} d E_{C^{*}}(\zeta)=Y
\end{aligned}
$$

by (4.28). It follows from (4.33) that $Y f \in \operatorname{Dom}\left(A^{*}\right)$ for any $f \in \operatorname{Dom}\left(C^{*}\right)$ and, thus,

$$
\operatorname{Ran}\left(\left.Y\right|_{\operatorname{Dom}\left(C^{*}\right)}\right) \subset \operatorname{Dom}\left(A^{*}\right) .
$$


Applying $A^{*}-\xi$ to both sides of the resulting equality (4.33) one infers that $Y$ is a strong solution to the dual Sylvester equation 4.10, which completes the proof.

Remark 4.6. Under the hypothesis of Theorem 4.5 the integral (4.9) converges in the sense of the strong operator topology if and only if so does the integral 4.11. This can be seen by combining Theorem 4.5 with Lemma 4.3 The operators $X$ and $Y$ given by the integrals (4.9) and (4.11) (if they exist in the sense of the strong operator topology) are related to each other by $Y=-X^{*}$

Lemma 4.7. Assume that the hypothesis of Theorem 4.5 holds. If at least one of the integrals (4.9) and 4.11) converges in the sense of the weak operator topology then both of them converge also in the sense of the strong operator topology.

Proof. Suppose that the integral (4.9) converges in the sense of the weak operator topology. Let

$$
X_{\Omega}=\int_{\operatorname{spec}(C) \cap \Omega} d E_{C}(\zeta) D(A-\zeta)^{-1},
$$

where $\Omega=[a, b) \times \mathrm{i}[c, d)$ is a finite rectangle in $\mathbb{C}$. By the same reasoning as in the proof of equality 4.21] one obtains

$$
X_{\Omega} A f-C X_{\Omega} f=E_{C}(\Omega) D f \text { for any } f \in \operatorname{Dom}(A),
$$

taking into account that $\operatorname{Ran}\left(X_{\Omega}\right) \subset \operatorname{Ran}\left(E_{C}(\Omega)\right)$ by Remark 2.8 and hence $X_{\Omega} f \in \operatorname{Dom}(C)$. From (4.36) it follows that

$$
\begin{gathered}
\left\langle X_{\Omega} A f, g\right\rangle-\left\langle X_{\Omega} f, C^{*} g\right\rangle=\left\langle E_{C}(\Omega) D f, g\right\rangle \\
\text { for all } f \in \operatorname{Dom}(A) \text { and } g \in \operatorname{Dom}\left(C^{*}\right) .
\end{gathered}
$$

Passing in 4.37) to the limit as $a, c \rightarrow-\infty$ and $b, d \rightarrow+\infty$ yields that $X$ given by the (improper) weak integral (4.9) is a weak solution to the Sylvester equation (4.1) since by the assumption $w-\lim X_{\Omega}=X$ and since $s-\lim E_{C}(\Omega)=I$. Then by Lemma4.2 the operator $X$ is a strong solution to 4.1) and hence Theorem 4.5 (ii) implies that the integral 4.9 converges in the sense of the strong operator topology. By Remark 4.6 one concludes that so does the integral 4.11 .

Under the assumption that the integral (4.11) converges in the sense of the weak operator, the assertion is proven in a similar way.

The next statement concerns sufficient conditions for the existence of a strong (and even operator) solution to the Sylvester equation. The statement is an extension of [4, Lemma 2.18].

Lemma 4.8. Assume hypothesis of Theorem 4.5 Suppose that the condition

$$
\sup _{\zeta \in \operatorname{spec}(C)}\left\|(A-\zeta)^{-1}\right\|<\infty
$$

holds and the operator $D$ has a finite $E_{C^{-}}$norm, that is,

$$
\|D\|_{E_{C}}<\infty .
$$

Then equations (4.1) and (4.6) have unique strong solutions $X \in \mathscr{B}(\mathscr{H}, \mathscr{K})$ given by 4.9] and $Y \in \mathscr{B}(\mathscr{K}, \mathscr{H})$ given by 4.11), respectively. Moreover, $Y=-X^{*}$ and the Stieltjes integrals (4.9) and (4.11) exist in the sense of the uniform operator topology.

Assume, in addition, that

$$
\sup _{\zeta \in \operatorname{spec}(C)}\left\|\zeta(A-\zeta)^{-1}\right\|<\infty .
$$


Then

$$
\begin{aligned}
& \operatorname{Ran}(X) \subset \operatorname{Dom}(C), \\
& \operatorname{Ran}(Y) \subset \operatorname{Dom}\left(A^{*}\right),
\end{aligned}
$$

and thus $X$ and $Z$ appear to be operator solutions to (4.1) and (4.6), respectively.

We skip the proof since it almost literally repeats the proof of Lemma 2.18 in [4]. The only difference is in extending the Stieltjes integration in the corresponding formulas from the real axis to the complex plane.

Remark 4.9. Assume that

$$
\delta=\operatorname{dist}(\mathscr{W}(A), \operatorname{spec}(C))>0,
$$

where $\mathscr{W}(A)$ denotes the numerical range of $A$. Then by [14] Lemma V.6.1] it follows from (4.9) and 4.39) that

$$
\|X\|_{E_{C}} \leq \frac{1}{\delta}\|D\|_{E_{C}}
$$

Remark 4.10. If $A$ is normal, then by 4.9 it immediately follows from (4.39) that

$$
\|X\|_{E_{C}} \leq \frac{1}{d}\|D\|_{E_{C}},
$$

where $d=\operatorname{dist}(\operatorname{spec}(A), \operatorname{spec}(C))$. In this case one can also represent the operator $X$ in the form of a double Stieltjes operator integral [7],

$$
X=\int_{\operatorname{spec}(C)} \int_{\operatorname{spec}(A)} d E_{C}(\zeta) \frac{D}{z-\zeta} d E_{A}(z)
$$

If $D \in \mathscr{B}_{2}(\mathfrak{H}, \mathfrak{K})$ then by $[8$ Theorem 1] the operator $X$ is also Hilbert-Schmidt and the following estimate holds

$$
\|X\|_{2} \leq \frac{1}{d}\|D\|_{2}
$$

which is sharp in the class of Hilbert-Schmidt operators.

\section{RICCATI EQUATION}

There are at least three approaches that allow to tackle the Riccati equations involving operators on infinite-dimensional Hilbert spaces. The first of these approaches, going back to C. Davis [12] and Halmos [15], is based on a deep connection between theory of Riccati equations and results on variation of invariant subspaces of an operator under perturbation. We refer to the recent publication [17] discussing this purely geometric approach and its present status in great detail. Here we only mention that such an approach is essentially restricted to the operator Riccati equations associated with self-adjoint block operator matrices, that is, to the case of (1.11) with $A=A^{*}, C=C^{*}$, and $D=B^{*}$. Notice that the sharp norm estimates for variation of spectral subspaces under a perturbation obtained in [13. 19. 26] imply the corresponding sharp norm estimates for solutions of the associated Riccati equations.

The other approach is based on the factorization theorems for holomorphic operatorvalued functions proven by Markus and Matsaev [21] and by Virozub and Matsaev [29]. Several existence results for operator Riccati equations have been obtained within this approach (see [18, 23]) including an existence result [20] for the case where the entries $A$ and $C$ are allowed to be non-self-adjoint operators.

The third approach [4, 22, 24] (see also [2] and [16]) is closely related to the integral representation (4.9) for the solution of the operator Sylvester equation in the form of an 
operator integral. Using this representation allows one to rewrite the Riccati equation in the form of an equivalent integral equation that admits an application of Banach's Fixed Point Principle. So far, only the Riccati equations 1.11 with at least one of the entries $A$ and $C$ being a self-adjoint operator were studied within such an approach. In this section we derive consequences of the integral representation (4.9) that work for more general Riccati equations (1.11), where one of the entries $A$ and $C$ is merely a normal operator.

First, we recall the concepts of weak, strong, and operator solutions to the operator Riccati equations.

Definition 5.1. Assume that $A$ and $C$ are possibly unbounded densely defined closed operators on the Hilbert spaces $\mathfrak{H}$ and $\mathfrak{K}$, respectively. Let $B$ and $D$ be bounded operators from $\mathfrak{K}$ to $\mathfrak{H}$ and from $\mathfrak{H}$ to $\mathfrak{K}$, respectively.

A bounded operator $X \in \mathscr{B}(\mathfrak{H}, \mathfrak{K})$ is said to be a weak solution of the Riccati equation

$$
X A-C X+X B X=D
$$

if

$$
\begin{gathered}
\langle X A f, g\rangle-\left\langle X f, C^{*} g\right\rangle+\langle X B X f, g\rangle=\langle D f, g\rangle \\
\text { for all } f \in \operatorname{Dom}(A) \text { and } g \in \operatorname{Dom}\left(C^{*}\right) .
\end{gathered}
$$

A bounded operator $X \in \mathscr{B}(\mathfrak{H}, \mathfrak{K})$ is called a strong solution of the Riccati equation (5.1) if

$$
\operatorname{Ran}\left(\left.X\right|_{\operatorname{Dom}(A)}\right) \subset \operatorname{Dom}(C),
$$

and

$$
X A f-C X f+X B X f=D f \text { for all } f \in \operatorname{Dom}(A) \text {. }
$$

Finally, $X \in \mathscr{B}(\mathfrak{H}, \mathfrak{K})$ is said to be an operator solution of the Riccati equation 5.1 if

$$
\operatorname{Ran}(X) \subset \operatorname{Dom}(C)
$$

the operator $X A$ is bounded on $\operatorname{Dom}(X A)=\operatorname{Dom}(A)$, and equality

$$
\overline{X A}-C X+X B X=D
$$

holds as an operator equality, where $\overline{X A}$ denotes the closure of $X A$.

Along with the Riccati equation 5.1 we also introduce the dual equation

$$
Y C^{*}-A^{*} Y+Y B^{*} Y=D^{*} \text {. }
$$

The following assertion is a corollary of Lemma 4.2

Lemma 5.2. Let $A$ and $C$ be densely defined possibly unbounded closed operators on the Hilbert spaces $\mathfrak{H}$ and $\mathfrak{K}$, respectively. If $X \in \mathscr{B}(\mathfrak{K}, \mathfrak{H})$ is a weak solution of the Riccati equation (5.1) then $X$ is also a strong solution of (5.1).

Proof. The assumption that $X$ is a weak solution to the Riccati equation (5.1) implies that $X$ is a weak solution to the Sylvester equation

$$
X \widetilde{A}-C X=D
$$

where

$$
\widetilde{A}=A+B X \text { with } \operatorname{Dom}(\widetilde{A})=\operatorname{Dom}(A)
$$

is a closed densely defined operator on $\mathfrak{H}$. Hence by Lemma 4.2 the operator $X$ is also a strong solution to (5.6), that is, $\operatorname{Ran}\left(\left.X\right|_{\operatorname{Dom}(\widetilde{A})}\right) \subset \operatorname{Dom}(C)$ and

$$
X \widetilde{A} f-C X f=D f \text { for all } f \in \operatorname{Dom}(\widetilde{A}) .
$$

Taking into account (5.7), one then concludes that $X$ is a strong solution to 5.1. 
The next statement is a direct corollary of Lemma 4.3

Lemma 5.3. Let $A$ and $C$ be densely defined possibly unbounded closed operators on the Hilbert spaces $\mathfrak{H}$ and $\mathfrak{K}$, respectively, and $B \in \mathscr{B}(\mathfrak{K}, \mathfrak{H}), D \in \mathscr{B}(\mathfrak{H}, \mathfrak{K})$. Then $X \in \mathscr{B}(\mathfrak{H}, \mathfrak{K})$ is a weak (and hence strong) solution to the Riccati equation (5.1) if and only if $Y=-X^{*}$ is a weak (and hence strong) solution to the dual Riccati equation (5.5).

Throughout the remaining part of the section we assume the following hypothesis.

Hypothesis 5.4. Assume that $\mathfrak{H}$ and $\mathfrak{K}$ are Hilbert spaces, $A$ is a possibly unbounded densely defined closed operator on $\mathfrak{H}$ and $C$ a normal operator on $\mathfrak{K}$. Also assume that $B \in \mathscr{B}(\mathfrak{K}, \mathfrak{H})$ and $D \in \mathscr{B}(\mathfrak{H}, \mathfrak{K})$.

The representation theorems of Sec. 4 for solutions of the Sylvester equation provide us with iteration schemes allowing one to prove solvability of the Riccati equations by using fixed point theorems.

Theorem 5.5. Assume Hypothesis 5.4 Then the following statements hold.

(i) Assume, in addition to Hypothesis 5.4 that

$$
\operatorname{dist}(\operatorname{spec}(A+B X), \operatorname{spec}(C))>0 \text {. }
$$

Then $X \in \mathscr{B}(\mathfrak{H}, \mathfrak{K})$ is a weak (and hence strong) solution to the Riccati equation (5.1) if and only if $X$ is a solution of the equation

$$
X=\int_{\operatorname{spec}(C)} d E_{C}(\zeta) D(A+B X-\zeta)^{-1}
$$

where the operator Stieltjes integral exists in the sense of the weak (and hence strong) operator topology in $\mathscr{B}(\mathfrak{H}, \mathfrak{K})$.

(ii) Assume, in addition to Hypothesis 5.4 that $Y \in \mathscr{B}(\mathfrak{K}, \mathfrak{H})$ and

$$
\operatorname{dist}\left(\operatorname{spec}\left(A^{*}-Y B^{*}\right), \operatorname{spec}\left(C^{*}\right)\right)>0 .
$$

Then the operator $Y$ is a weak (and hence strong) solution to the dual Riccati equation (5.5) if and only if $Y$ satisfies the equation

$$
Y=-\int_{\operatorname{spec}\left(C^{*}\right)}\left(A^{*}-Y B^{*}-\zeta\right)^{-1} D^{*} d E_{C^{*}}(\zeta)
$$

where the operator Stieltjes integral exists in the sense of the weak (and hence strong) operator topology.

Proof. ( $i$ ) The operator $X$ is a weak (and hence strong) solution to 5.1 if and only if $X$ is a weak (and hence strong) solution to the equation

$$
X \widetilde{A}-C X=D
$$

where

$$
\widetilde{A}=A+B X .
$$

Applying Theorem 4.5 $(i)$ and Lemma 4.7 completes the proof of $(i)$.

(ii) The operator $Y$ is a weak (and hence strong) solution to 5.5 if and only if $Y$ is a weak (and hence strong) solution to the equation

$$
Y C-\widehat{A} Y=D^{*}
$$

where

$$
\widehat{A}=A-Y B^{*} .
$$

Applying Theorem 4.5 (ii) and Lemma 4.7 completes the proof of $(i i)$.

The proof is complete. 
The following statement is a direct corollary of Lemma 4.8

Theorem 5.6. Assume Hypothesis 5.4 and let $D$ have a finite norm with respect to the spectral measure of the normal operator $C$, that is,

$$
\|D\|_{E_{C}}<\infty \text {. }
$$

Assume, in addition, that a bounded operator $X$ from $\mathfrak{H}$ to $\mathfrak{K}$ is a weak solution of the Riccati equation (5.1) such that

$$
\operatorname{dist}(\operatorname{spec}(A+B X), \operatorname{spec}(C))>0,
$$

and that the condition

$$
\sup _{\zeta \in \operatorname{spec}(C)}\left\|(A+B X-\zeta)^{-1}\right\|<\infty
$$

holds.

Then $X$ is a strong solution to (5.1) and the operator $Y=-X^{*}$ is a strong solution to the dual Riccati equation 5.5.

The strong solutions $X$ and $Y$ admit the representations

$$
\begin{aligned}
& X=\int_{\operatorname{spec}(C)} d E_{C}(\zeta) D(A+B X-\zeta)^{-1}, \\
& Y=-\int_{\operatorname{spec}(C)}\left(A-Y B^{*}-\zeta\right)^{-1} D^{*} d E_{C^{*}}(\zeta),
\end{aligned}
$$

where the operator Stieltjes integrals exist in the sense of the uniform operator topology. Hence, the operators $X$ and $Y$ have finite $E_{C}-$ norm and the following bound holds true

$$
\|Y\|_{E_{C}}=\|X\|_{E_{C}} \leq\|D\|_{E_{C}} \sup _{\zeta \in \operatorname{spec}(C)}\left\|(A+B X-\zeta)^{-1}\right\| .
$$

If, in this case, instead of (5.14 the following condition holds

$$
\sup _{\zeta \in \operatorname{spec}(C)}\left\|\zeta(A+B X-\zeta)^{-1}\right\|<\infty,
$$

then

$$
\operatorname{Ran}(X) \subset \operatorname{Dom}(C), \quad \operatorname{Ran}(Y) \subset \operatorname{Dom}\left(A^{*}\right)
$$

and, hence, the strong solutions $X$ and $Y$ appear to be operator solutions to the Riccati equations (5.1) and (5.5), respectively.

In the case where the spectrum of the normal operator $C$ is separated from the numerical range $\mathscr{W}(A)$ of the operator $A$ we are able to prove the existence of a fixed point for the mapping (5.9), provided that the operators $B$ and $D$ satisfy certain "smallness" assumptions. If, in addition, $A$ is also a normal operator, we prove the existence of such a fixed point under weaker assumptions.

Theorem 5.7. Under Hypothesis 5.4 assume that $B \neq 0$ and either

$$
\text { (i) } d=\operatorname{dist}(\mathscr{W}(A), \operatorname{spec}(C))>0
$$

or

(ii) A is a normal operator on $\mathfrak{H}$ and

$$
d=\operatorname{dist}(\operatorname{spec}(A), \operatorname{spec}(C))>0 .
$$

Also assume that the operator D has a finite $E_{C}-$ norm and

$$
\sqrt{\|B\|\|D\|_{E_{C}}}<\frac{d}{2}
$$


Then the Riccati equation (5.1) has a unique strong solution in the ball

$$
\left\{X \in \mathscr{B}(\mathfrak{H}, \mathfrak{K}) \mid\|X\|<\|B\|^{-1}\left(d-\sqrt{\|B\|\|D\|_{E_{C}}}\right)\right\} .
$$

Moreover, the strong solution $X$ has a finite $E_{C}-n o r m$ that satisfies the bound

$$
\|X\|_{E_{C}} \leq \frac{1}{\|B\|}\left(\frac{d}{2}-\sqrt{\frac{d^{2}}{4}-\|B\|\|D\|_{E_{C}}}\right) .
$$

In particular, for

or

$$
\|B\|<\frac{d}{2} \text { and }\|B\|+\|D\|_{E_{C}}<d
$$

$$
\|B\| \geq \frac{d}{2}\left(\text { and }\|D\|_{E_{C}}<\frac{d^{2}}{4\|B\|}\right)
$$

the strong solution $X$ is a strict contraction in both the $E_{C}$-norm and uniform operator topologies,

$$
\|X\| \leq\|X\|_{E_{C}}<1 .
$$

Proof. Technically, the proof of the assertion is very similar to that of the second part of Theorem 3.6 in [4] and it follows the same line in both cases $(i)$ and $(i i)$. Therefore we present here only the proof for the case $(i)$.

Assume the hypothesis with assumption $(i)$.

We notice, first, that under this assumption the resolvent $(A-\zeta)^{-1}$ for $\zeta \in \operatorname{spec}(C)$ is a bounded operator since $\operatorname{spec}(A) \subset \overline{\mathscr{W}(A)}$. Moreover, by [14 Lemma V.6.1] the following inequality holds

which 5.19 yields

$$
\left\|(A-\zeta)^{-1}\right\| \leq \frac{1}{\operatorname{dist}(\zeta, \mathscr{W}(A))},
$$

$$
\sup _{\zeta \in \operatorname{spec}(C)}\left\|(A-\zeta)^{-1}\right\| \leq \frac{1}{d}
$$

Given $r \in(0, d /\|B\|)$, denote by $\mathscr{O}_{r}$ the closed $r$-neighbourhood of the zero operator in $\mathscr{B}(\mathfrak{H}, \mathfrak{K})$, i.e. $\mathscr{O}_{r}=\{X \in \mathscr{B}(\mathfrak{H}, \mathfrak{K}) \mid\|X\| \leq r\}$. Clearly, for any $X \in \mathscr{O}_{r}$ by [5.27) we have

$$
\begin{aligned}
\sup _{\zeta \in \operatorname{spec}(C)}\left\|\left(I+(A-\zeta)^{-1} B X\right)^{-1}\right\| & \leq \sup _{\zeta \in \operatorname{spec}(C)} \frac{1}{1-\left\|(A-\zeta)^{-1}\right\|\|B\|\|X\|} \\
& \leq \frac{1}{1-\frac{\|B\| r}{d}}
\end{aligned}
$$

Therefore, the inverse operators involved in the identity

$$
\begin{aligned}
(A+B X-\zeta)^{-1}= & \left(I+(A-\zeta)^{-1} B X\right)^{-1}(A-\zeta)^{-1}, \\
& \zeta \in \operatorname{spec}(C), X \in \mathscr{O}_{r},
\end{aligned}
$$

are well defined. By (5.27) and 5.28) from (5.29) it follows that

$$
\sup _{\zeta \in \operatorname{spec}(C)}\left\|(A+B X-\zeta)^{-1}\right\| \leq \frac{1}{d-\|B\| r}, \text { whenever } X \in \mathscr{O}_{r} .
$$

Since (5.30) holds and the operator $D$ has a finite $E_{C}$-norm, the mapping

$$
F(X)=\int_{\operatorname{spec}(C)} d E_{C}(\zeta) D(A+B X-\zeta)^{-1}
$$


from $\mathscr{B}(\mathfrak{H})$ to $\mathscr{B}(\mathfrak{K})$ is well defined on $\operatorname{Dom}(F)=\mathscr{O}_{r}$. Notice that by Lemma 3.2 the operator Stieltjes integral in 5.31) exists even in the sense of the uniform operator topolgy.

The assumption that $X \in \mathscr{O}_{r}$ implies that the numerical range $\mathscr{W}(A+B X)$ of the operator $A+B X$ lies in the closed $(\|B\| r)$-neighborhood of $\mathscr{W}(A)$. Since $\|B\| r<d$ and $\operatorname{spec}(A+$ $B X) \subset \overline{\mathscr{W}(A+B X)}$, one then concludes that dist $(\operatorname{spec}(A+B X), \operatorname{spec}(C)) \geq d-\|B\| r>0$. Hence from Theorem 5.5 $(i)$ it follows that any fixed point of the mapping $F$ in the ball $\mathscr{O}_{r}$, if it exists, appears to be a strong solution to the Riccati equation 5.1.

Using (5.30) we obtain the following two estimates

$$
\begin{aligned}
\|F(X)\| \leq\|F(X)\|_{E_{C}} & \leq\|D\|_{E_{C}} \sup _{\zeta \in \operatorname{spec}(C)}\left\|(A+B X-\zeta)^{-1}\right\| \\
& \leq \frac{\|D\|_{E_{C}}}{d-\|B\| r}, \quad X \in \mathscr{O}_{r}
\end{aligned}
$$

and

$$
\begin{aligned}
\| F\left(X_{1}\right) & -F\left(X_{2}\right) \| \\
\leq & \left\|F\left(X_{1}\right)-F\left(X_{2}\right)\right\|_{E_{C}} \\
\quad & \left\|\int_{\operatorname{spec}(C)} d E_{C}(\zeta) D\left(A+B X_{1}-\zeta\right)^{-1} B\left(X_{2}-X_{1}\right)\left(A+B X_{2}-\zeta\right)^{-1}\right\|_{E_{C}} \\
& \leq \frac{\|B\|\|D\|_{E_{C}}}{(d-\|B\| r)^{2}}\left\|X_{2}-X_{1}\right\|, \quad X_{1}, X_{2} \in \mathscr{O}_{r} .
\end{aligned}
$$

Inequality (5.32) implies that $F$ maps the ball $\mathscr{O}_{r}$ into itself whenever

$$
\frac{\|D\|_{E_{C}}}{d-\|B\| r} \leq r
$$

By (5.33) it follows that $F$ is a strict contraction on $\mathscr{O}_{r}$ if

$$
\frac{\|B\|\|D\|_{E_{C}}}{(d-\|B\| r)^{2}}<1 .
$$

Solving (5.34) and (5.35) with respect to $r$ simultaneously, one obtains that if the radius of the ball $\mathscr{O}_{r}$ is within the bounds

$$
\frac{1}{\|B\|}\left(\frac{d}{2}-\sqrt{\frac{d^{2}}{4}-\|B\|\|D\|_{E_{C}}}\right) \leq r<\frac{1}{\|B\|}\left(d-\sqrt{\|B\|\|D\|_{E_{C}}}\right),
$$

then $F$ is a strictly contracting mapping of the ball $\mathscr{O}_{r}$ into itself. Applying Banach's Fixed Point Theorem one then infers that equation (5.9) has a unique solution in any ball $\mathscr{O}_{r}$ whenever $r$ satisfies 5.36 . Therefore, the fixed point does not depend on the radii satisfying (5.36) and hence it belongs to the smallest of these balls. This observation proves the estimate

$$
\|X\| \leq \frac{1}{\|B\|}\left(\frac{d}{2}-\sqrt{\frac{d^{2}}{4}-\|B\|\|D\|_{E_{C}}}\right) .
$$

Finally, using [5.32, for the fixed point $X$ one obtains the estimate

$$
\|X\|_{E_{C}}=\|F(X)\|_{E_{C}} \leq \frac{\|D\|_{E_{C}}}{d-\|B\|\|X\|} .
$$


Then 5.37 yields

$$
\|X\|_{E_{C}} \leq \frac{\|D\|_{E_{C}}}{\frac{d}{2}+\sqrt{\frac{d^{2}}{4}-\|B\|\|D\|_{E_{C}}}}=\frac{1}{\|B\|}\left(\frac{d}{2}-\sqrt{\frac{d^{2}}{4}-\|B\|\|D\|_{E_{C}}}\right),
$$

proving (5.23).

Finally, using 5.21 and (5.23) one easily verifies by inspection that any of the assumptions (5.24) and (5.25) implies (5.26).

The proof is complete.

Remark 5.8. This theorem extends results obtained in [4, 22, 24, 25] where the operators $A$ and $C$ are assumed to be self-adjoint. In case where the self-adjoint operator $C$ is bounded, $D$ is a Hilbert-Schmidt operator, $B$ is bounded, and $A$ is possibly unbounded densely defined closed non-self-adjoint operator, the solvability of the equation (5.15) under condition [5.20] has also been studied in [2].

Acknowledgments. This was supported by the Deutsche Forschungsgemeinschaft (DFG), the Heisenberg-Landau Program, and the Russian Foundation for Basic Research. A. K. Motovilov gratefully acknowledges the kind hospitality of the Institut für Angewandte Mathematik, Universität Bonn.

\section{REFERENCES}

[1] V. M. Adamyan, H. Langer, R. Mennicken, and J. Saurer, Spectral components of selfadjoint block operator matrices with unbounded entries, Math. Nachr. 178 (1996), 43-80.

[2] V. Adamyan, H. Langer, and C. Tretter, Existence and uniqueness of contractive solutions of some Riccati equations, J. Funct. Anal. 179 (2001), 448-473.

[3] N. I. Akhiezer and I. M. Glazman, Theory of linear operators in Hilbert space, Dover Publications Inc., New York, 1993.

[4] S. Albeverio, K. A. Makarov, and A. K. Motovilov, Graph subspaces and the spectral shift function, Can. J. Math., 55:3 (2003), 449 - 503; arXiv: math.SP/0105142 v3.

[5] R. Bhatia and P. Rosenthal, How and why to solve the operator equation $A X-X B=Y$, Bull. London Math. Soc. 29 (1997), 1 - 21.

[6] M. Sh. Birman and M.Z. Solomjak, Spectral Theory of Self-Adjoint Operators on a Hilbert Space, Izdat. Leningrad. Univ., Leningrad, 1980 (Russian); English translation: D. Reidel Publishing Company, Dordrecht, 1986.

[7] M. Sh. Birman and M. Solomyak, Double operator integrals in a Hilbert space, Integr. Eq. Oper. Th. 47 (2003), $131-168$.

[8] M. Sh. Birman and M.Z. Solomyak, Stieltjes double-operator integrals, Topics in Mathematical Physics, Vol. 1, Consultants Bureau, New York, 1967, pp. 25 - 54.

[9] M. Sh. Birman and M. Z. Solomyak, Double Stieltjes operator integrals. II, Topics in Mathematical Physics, Vol. 2, Consultants Bureau, New York, 1968, pp. $19-46$.

[10] M. Sh. Birman and M.Z. Solomyak, Double Stieltjes operator integrals. III, Problems of mathematical physics. No. 6. Theory of functions. Spectral theory. Wave propagation, Izdat. Leningrad. Univ., Leningrad, 1973, pp. 27 - 53 (Russian).

[11] Y. Daleckii, On the asymptotic solution of a vector differential equation, Dokl. Akad. Nauk SSSR 92 (1953), $881-884$.

[12] C. Davis, Separation of two linear subspaces, Acta Scient. Math. (Szeged) 19 (1958), 172 - 187.

[13] C. Davis and W. M. Kahan, The rotation of eigenvectors by a perturbation. III, SIAM J. Numer. Anal. 7 (1970), $1-46$.

[14] I. C. Gohberg and M. G. Krein, Introduction to the Theory of Linear Non-Selfadjoint Operators, Translations of Mathematical Monographs, vol. 18, American Mathematical Society, Providence, 1969.

[15] P. R. Halmos, Two subspaces, Trans. Amer. Math. Soc. 144 (1969), 381 - 389.

[16] V. Hardt, R. Mennicken, and A. K. Motovilov, Factorization theorem for the transfer function associated with a $2 \times 2$ operator matrix having unbounded couplings, J. Oper. Th. 48 No. 1 (2002), 187 - 226. 
[17] V. Kostrykin, K. A. Makarov, and A. K. Motovilov, Existence and uniqueness of solutions to the operator Riccati equation. A geometric approach, Contemporary Mathematics (AMS) 327 (2003), 181 - 198; arXiv: math.SP/0207125

[18] V. Kostrykin, K. A. Makarov, and A. K. Motovilov, On the existence of solutions to the operator Riccati equation and the $\tan \Theta$ theorem, Int. Eq. Op. Th. 51 (2005), 121-140; arXiv: math.SP/0210032 v2.

[19] V. Kostrykin, K. A. Makarov, and A. K. Motovilov, Perturbation of spectra and spectral subspaces, Tran. Amer. Math. Soc. (to appear); arXiv: math.SP/0306025 v1.

[20] H. Langer, A. Markus, V. Matsaev, and C. Tretter, A new concept for block operator matrices: the quadratic numerical range, Linear Algebra Appl. 330 (2001), 89 - 112.

[21] A. S. Markus and V. I. Matsaev On the spectral theory of holomorphic operator-valued functions in Hilbert space, Funct. Anal. Appl. 9 (1975) No. 1, 73 - 74.

[22] R. Mennicken and A. K. Motovilov, Operator interpretation of resonances arising in spectral problems for $2 \times 2$ operator matrices, Math. Nachr. 201 (1999), 117 - 181; arXiv: funct-an/9708001

[23] R. Mennicken and A. A. Shkalikov, Spectral decomposition of symmetric operator matrices, Math. Nachr. 179 (1996), $259-273$.

[24] A. K. Motovilov, Potentials appearing after removal of the energy-dependence and scattering by them, In: Proc. of the Intern. Workshop "Mathematical aspects of the scattering theory and applications", St. Petersburg University, St. Petersburg, 1991, pp. $101-108$.

[25] A. K. Motovilov, Removal of the resolvent-like energy dependence from interactions and invariant subspaces of a total Hamiltonian, J. Math. Phys. 36 (1995), 6647 - 6664; arXiv:funct-an/9606002

[26] A. K. Motovilov and A. V. Selin, Some sharp norm estimates in the subspace perturbation problem, arXiv: math.SP/0409558

[27] V. Q. Phóng, The operator equation $A X-X B=C$ with unbounded operators $A$ and $B$ and related abstract Cauchy problems, Math. Z. 208 (1991), $567-588$.

[28] M. Rosenblum, On the operator equation $B X-X A=Q$, Duke Math. J. 23 (1956), $263-269$.

[29] A.I. Virozub and V. I. Matsaev, The spectral properties of a certain class of selfadjoint operator functions, Funct. Anal. Appl. 8 (1974), 1 - 9.

Sergio Albeverio, Institut für Angewandte Mathematik, Universität Bonn, WegelerStrasse 6, D-53115 Bonn, Germany; SFB 611, BonN; BiBoS, Bielefeld-Bonn; CERFIM, LoCARNo; ACCADEMIa Di ARChitetTURA, USI, MENDRISIO

E-mail address: albeverio@uni-bonn.de

Alexander K. Motovilov, Bogoliubov laboratory of Theoretical Physics, JinR, JoliotCuRIE 6, 141980 Dubna, Moscow Region, Russia

E-mail address: motovilv@theor.jinr.ru 